\title{
Article \\ Some New Beesack-Wirtinger-Type Inequalities Pertaining to Different Kinds of Convex Functions
}

\author{
Artion Kashuri $^{1}$ (D), Muhammad Samraiz ${ }^{2}$ (D), Gauhar Rahman ${ }^{3, *(D)}$ and Zareen A. Khan ${ }^{4, *(D)}$ \\ 1 Department of Mathematics, Faculty of Technical and Natural Science, University "Ismail Qemali", \\ 9400 Vlora, Albania; artion.kashuri@univlora.edu.al or artionkashuri@gmail.com \\ 2 Department of Mathematics, University of Sargodha, Sargodha 40100, Pakistan; \\ muhammad.samraiz@uos.edu.pk or msamraizuos@gmail.com \\ 3 Department of Mathematics and Statistics, Hazara University, Mansehra 21300, Pakistan \\ 4 Department of Mathematical Sciences, College of Science, Princess Nourah Bint Abdulrahman University, \\ P.O. Box 84428, Riyadh 11671, Saudi Arabia \\ * Correspondence: gauhar55uom@gmail.com or drgauhar.rahman@hu.edu.pk (G.R.); \\ zakhan@pnu.edu.sa (Z.A.K.)
}

check for

updates

Citation: Kashuri, A.; Samraiz, M.; Rahman, G.; Khan, Z.A. Some New Beesack-Wirtinger-Type Inequalities Pertaining to Different Kinds of Convex Functions. Mathematics 2022, 10, 757. https://doi.org/10.3390/ math10050757

Academic Editors: Ana Vukelic and Shin-ya Matsushita

Received: 15 December 2021 Accepted: 24 February 2022

Published: 27 February 2022

Publisher's Note: MDPI stays neutral with regard to jurisdictional claims in published maps and institutional affiliations.

Copyright: (C) 2022 by the authors Licensee MDPI, Basel, Switzerland. This article is an open access article distributed under the terms and conditions of the Creative Commons Attribution (CC BY) license (https:// creativecommons.org/licenses/by/ $4.0 /)$.
Abstract: In this paper, the authors established several new inequalities of the Beesack-Wirtinger type for different kinds of differentiable convex functions. Furthermore, we generalized our results for functions that are $n$-times differentiable convex. Finally, many interesting Ostrowski- and Chebyshevtype inequalities are given as well.

Keywords: Wirtinger inequality; Beesack inequality; Chebyshev inequality; Ostrowski inequality; Hölder inequality; convexity

MSC: Primary: 26A51; Secondary: 26A33; 26D07; 26D10; 26D15

\section{Introduction and Preliminaries}

The following inequality regarding square integrable functions is known as the Wirtinger inequality:

Theorem 1 ([1,2]). Let $\omega$ be a real-valued function with period $2 \pi$ and $\int_{0}^{2 \pi} \omega(\mu) d \mu=0$. If $\omega^{\prime} \in L^{2}[0,2 \pi]$, then:

$$
\int_{0}^{2 \pi}[\omega(\mu)]^{2} d \mu \leq \int_{0}^{2 \pi}\left[\omega^{\prime}(\mu)\right]^{2} d \mu
$$

with equality holding iff $\omega(\mu)=\mathcal{A}_{1} \cos \mu+\mathcal{A}_{2} \sin \mu$, where $\mathcal{A}_{1}, \mathcal{A}_{2} \in \mathbb{R}$. For recently published papers of this type, see [3-5].

Beesack in [6,7] generalized (1) as follows:

Theorem 2. Let $\omega$ be absolutely continuous on $\left[0, \frac{\pi}{2}\right)$ with $\omega(0)=0$, then for all $p>1$, we have:

$$
\int_{0}^{2 \pi}[\omega(\mu)]^{p} d \mu \leq \frac{1}{p-1} \cdot\left[\frac{\pi}{2} \sin \left(\frac{\pi}{p}\right)\right]^{p} \int_{0}^{2 \pi}\left[\omega^{\prime}(\mu)\right]^{p} d \mu,
$$

with equality holding iff $\omega(\mu)=c \zeta(\mu)$ and $\zeta(\mu)$ satisfies the following equation:

$$
\mu=\frac{p}{2} \sin \left(\frac{\pi}{p}\right) \int_{0}^{\zeta} \frac{1}{\sqrt[p]{1-\delta^{p}}} d \delta, \quad 0 \leq \zeta \leq 1 .
$$


The next functional is known as the Chebyshev functional (see [8]):

$$
\mathcal{T}(\omega, \vartheta):=\frac{1}{\iota_{2}-\iota_{1}} \int_{\iota_{1}}^{\iota_{2}} \omega(\delta) \vartheta(\delta) d \delta-\frac{1}{\iota_{2}-\iota_{1}} \int_{\iota_{1}}^{\iota_{2}} \omega(\delta) d \delta \cdot \frac{1}{\iota_{2}-\iota_{1}} \int_{\iota_{1}}^{\iota_{2}} \vartheta(\delta) d \delta .
$$

Several bounds for $\mathcal{T}(\omega, \vartheta)$ have been found by many authors, and many important applications have been given. For example, Alomari in [9] obtained a bound for the Chebyshev functional. Maširević et al. in [10] established new bounds on the Chebyshev functional for the $C_{\varphi}[0,1]$ function class. Rahman et al. in [11] derived certain new proportional and Hadamard proportional fractional integral inequalities. Khan et al. in [12] investigated the Hirota equation using the modified double Laplace decomposition method. Rahman et al. in [13] obtained the weighted fractional integral inequalities for Chebyshev functionals. Khan et al. in [14] established applications of the fixed-point theory to investigate a system of factional-order differential equations. Ayub et al. in [15] used new a Mittag-Leffler function and derived its applications. Iqbal et al. in [16] found new generalized Pólya-Szegö- and Chebyshev-type inequalities with a general kernel and measure. Gul et al. in [17] investigated a class of boundary-value problems under the ABC fractional derivative. Nisar et al. in [18] derived the weighted fractional Pólya-Szegö- and Chebyshev-type integral inequalities concerning another function. Khan et al. in [19] investigated the impulsive boundary-value problem with the Riemann-Liouville fractional-order derivative. Rahman et al. in [20] established generalized fractional integral inequalities for the monotone weighted Chebyshev functionals. Srivastava et al. in [21] obtained new Chebyshev-type inequalities via a general family of fractional integral operators with a modified Mittag-Leffler kernel. Set et al. in [22] found Chebyshev-type inequalities by using generalized proportional Hadamard fractional integrals via the Polya-Szegö inequality with applications. Özdemir et al. in [23] obtained some new Chebyshev-type inequalities for functions whose derivatives belong to $L_{p}$ spaces. Akdemir et al. in [24] found new general variants of Chebyshev-type inequalities via generalized fractional integral operators. Butt et al. in [25] used Caputo fractional derivatives via exponential s-convex functions.

The following important results were obtained by Alomari in [9].

Lemma 1. Let $\iota_{1}<\iota_{2}$ and $\iota_{1}, \iota_{2} \in I^{\circ}$ (the interior set of I). Assume that $\omega$ is an absolutely continuous function on $I$, where $\omega$ and $\boldsymbol{\omega}^{\prime}$ are positive and $\omega\left(\iota_{1}\right)=0$. If $p>1$ and $\int_{\iota_{1}}^{\iota_{2}}\left[\mathcal{\omega}^{\prime}(\mu)\right]^{p} d \mu<\infty$, then:

$$
\int_{\iota_{1}}^{\iota_{2}}[\omega(\mu)]^{p} d \mu \leq \frac{p^{p} \sin ^{p}\left(\frac{\pi}{p}\right)}{\pi^{p}(p-1)}\left(\iota_{2}-\iota_{1}\right)^{p} \int_{\iota_{1}}^{\iota_{2}}\left[\omega^{\prime}(\mu)\right]^{p} d \mu .
$$

The constant $\frac{p^{p} \sin ^{p}\left(\frac{\pi}{p}\right)}{\pi^{p}(p-1)}\left(\iota_{2}-\iota_{1}\right)^{p}$ is the best possible for every $p>1$.

Lemma 2. Let $\iota_{1}<\iota_{2}$ and $\iota_{1}, \iota_{2} \in I^{\circ}$. Suppose that $\omega$ is an absolutely continuous function on $I$, where $\omega$ and $\omega^{\prime}$ are positive and $\omega\left(\iota_{2}\right)=0$. If $p>1$ and $\int_{\iota_{1}}^{\iota_{2}}\left[\omega^{\prime}(\mu)\right]^{p} d \mu<\infty$, then the inequality (4) holds.

Let us denote $\mathfrak{U}^{p}\left(\iota_{1}, \iota_{2}\right)$ where $p>1$ the space of all positive $n$-th differentiable functions $\omega$ whose $n$-th derivatives $\omega^{(n)}$ are positive locally absolutely continuous on $\left(\iota_{1}, \iota_{2}\right)$ with the condition that $\int_{\iota_{1}}^{\iota_{2}}\left[\omega^{(n)}(\mu)\right]^{p} d \mu<\infty$. Then, the above Lemmas 1 and 2 are generalized as follows: 
Lemma 3. Let $-\infty<\iota_{1}<\iota_{2}<\infty$. If $\mathfrak{\omega} \in \mathfrak{U}^{p}\left(\iota_{1}, \iota_{2}\right)$ with $\mathfrak{\omega}^{(j)}\left(\iota_{1}\right)=0$, for all $j=0,1, \ldots, n-1$, and $\int_{\iota_{1}}^{\iota_{2}}\left[\omega^{(n)}(\mu)\right]^{p} d \mu<\infty$, then for all $p>1$, we have:

$$
\int_{\iota_{1}}^{\iota_{2}}[\omega(\mu)]^{p} d \mu \leq\left(\frac{p^{p} \sin ^{p}\left(\frac{\pi}{p}\right)}{\pi^{p}(p-1)}\right)^{n}\left(\iota_{2}-\iota_{1}\right)^{n p} \int_{\iota_{1}}^{\iota_{2}}\left[\omega^{(n)}(\mu)\right]^{p} d \mu .
$$

Lemma 4. Let $-\infty<\iota_{1}<\iota_{2}<\infty$. If $\mathfrak{\omega} \in \mathfrak{U}^{p}\left(\iota_{1}, \iota_{2}\right)$ with $\omega^{(j)}\left(\iota_{2}\right)=0$, for all $j=0,1, \ldots, n-1$, and $\int_{\iota_{1}}^{\iota_{2}}\left[\mathcal{O}^{(n)}(\mu)\right]^{p} d \mu<\infty$, then for all $p>1$, the inequality (5) holds.

Furthermore, we define $\mathfrak{L}^{p}\left(\iota_{1}, \iota_{2}\right)$ with $p>1$ as the space of all positive differentiable functions $\omega$ whose first derivatives $\omega^{\prime}$ are positive locally absolutely continuous on $\left(\iota_{1}, \iota_{2}\right)$ and $\int_{l_{1}}^{\iota_{2}}\left[\omega^{\prime}(\mu)\right]^{p} d \mu<\infty$.

Theorem 3. Let $\xi \in\left(\iota_{1}, l_{2}\right)$, then for all $\omega \in \mathfrak{L}^{p}\left(\iota_{1}, l_{2}\right)$, we have:

$$
\int_{\iota_{1}}^{\iota_{2}}|\omega(\mu)-\omega(\xi)|^{p} d \mu \leq \frac{p^{p} \sin ^{p}\left(\frac{\pi}{p}\right)}{\pi^{p}(p-1)}\left[\frac{\iota_{2}-\iota_{1}}{2}+\left|\xi-\frac{\iota_{1}+\iota_{2}}{2}\right|\right]^{p} \cdot \int_{\iota_{1}}^{\iota_{2}}\left[\omega^{\prime}(\mu)\right]^{p} d \mu .
$$

Theorem 4. Let $\xi \in\left(\iota_{1}, \iota_{2}\right)$, then for all $\mathfrak{\omega} \in \mathfrak{U}^{p}\left(\iota_{1}, \iota_{2}\right)$, we obtain:

$$
\int_{\iota_{1}}^{\iota_{2}}|\omega(\mu)-\omega(\xi)|^{p} d \mu \leq\left(\frac{p^{p} \sin ^{p}\left(\frac{\pi}{p}\right)}{\pi^{p}(p-1)}\right)^{n}\left[\frac{\iota_{2}-\iota_{1}}{2}+\left|\xi-\frac{\iota_{1}+\iota_{2}}{2}\right|\right]^{n p} \cdot \int_{\iota_{1}}^{\iota_{2}}\left[\omega^{(n)}(\mu)\right]^{p} d \mu .
$$

The theory of convexity has played very important role in the development of the theory of inequalities. A wide class of inequalities can easily be obtained using the convexity property of the functions.

Let us recall the following definitions that are used in the sequel.

Definition 1 ([26]). A function $\omega: I \rightarrow \mathbb{R}$ is said to be convex, if:

$$
\omega\left(\delta \iota_{1}+(1-\delta) \iota_{2}\right) \leq \delta \omega\left(\iota_{1}\right)+(1-\delta) \omega\left(\iota_{2}\right), \quad \forall \iota_{1}, \iota_{2} \in I, \delta \in[0,1] .
$$

Definition 2 ([9]). A function $\omega: I \rightarrow \mathbb{R}$ is said to be P-convex, if:

$$
\omega\left(\delta \iota_{1}+(1-\delta) \iota_{2}\right) \leq \mathscr{\omega}\left(\iota_{1}\right)+\mathscr{\omega}\left(\iota_{2}\right), \quad \forall \iota_{1}, \iota_{2} \in I, \delta \in[0,1] .
$$

Definition 3 ([26]). A function $\omega: I \rightarrow \mathbb{R}$ is said to be quasi-convex, if:

$$
\omega\left(\delta \iota_{1}+(1-\delta) \iota_{2}\right) \leq \max \left\{\omega\left(\iota_{1}\right), \omega\left(\iota_{2}\right)\right\}, \quad \forall \iota_{1}, \iota_{2} \in I, \delta \in[0,1] .
$$

Definition 4 ([27]). A function $\omega: I \rightarrow \mathbb{R}$ is said to be s-convex for some fixed $s \in(0,1]$, if:

$$
\omega\left(\delta \iota_{1}+(1-\delta) \iota_{2}\right) \leq \delta^{S} \omega\left(\iota_{1}\right)+(1-\delta)^{S} \oplus\left(\iota_{2}\right), \quad \forall \iota_{1}, \iota_{2} \in I, \delta \in[0,1] .
$$

Definition 5 ([28,29]). A function $\omega:[0, l] \rightarrow \mathbb{R}$ is said to be $m$-convex for some fixed $m \in(0,1], i f:$

$$
\omega\left(\delta \iota_{1}+m(1-\delta) \iota_{2}\right) \leq \delta \omega\left(\iota_{1}\right)+m(1-\delta) \omega\left(\iota_{2}\right), \quad \forall \iota_{1}, \iota_{2} \in[0, \iota], \delta \in[0,1] .
$$


Definition 6 ([28,29]). A function $\omega:[0, \iota] \rightarrow \mathbb{R}$ is said to be $(\alpha, m)$-convex for some fixed $(\alpha, m) \in(0,1]^{2}$, if:

$$
\omega\left(\delta \iota_{1}+m(1-\delta) \iota_{2}\right) \leq \delta^{\alpha} \omega\left(\iota_{1}\right)+m\left(1-\delta^{\alpha}\right) \omega\left(\iota_{2}\right), \quad \forall \iota_{1}, \iota_{2} \in[0, \iota], \delta \in[0,1] .
$$

Motivated by the above results, the aim of this paper was to derive some new inequalities of the Beesack-Wirtinger type for different kinds of differentiable convex functions. Furthermore, we generalized our results for functions that are $n$-times differentiable convex. Finally, many interesting Ostrowski- and Chebyshev-type inequalities are given. Some conclusions and future research are provided as well. We hope that the ideas and techniques of this paper will inspire interested readers working in this fascinating field.

\section{Main Results}

In this main section, by applying Lemmas $1-4$, Theorems 3 and 4 , and the fact that every convex function is absolutely continuous, we derive the following inequalities of the Beesack-Wirtinger type.

Theorem 5. Let $\iota_{1}<\iota_{2}$ and $\iota_{1}, \iota_{2} \in I^{\circ}$. Assume that $\omega$ is a differentiable function on $I$, where $\omega$ and $\omega^{\prime}$ are positive and $\omega\left(\iota_{1}\right)=0$. If $\left[\omega^{\prime}(\mu)\right]^{p}$ is a P-convex function on $\left(\iota_{1}, \iota_{2}\right)$, then for all $p>1$, we have:

$$
\int_{\iota_{1}}^{\iota_{2}}[\omega(\mu)]^{p} d \mu \leq \mathcal{C}\left(p ; \iota_{1}, \iota_{2}\right)\left\{\left[\omega^{\prime}\left(\iota_{1}\right)\right]^{p}+\left[\omega^{\prime}\left(\iota_{2}\right)\right]^{p}\right\},
$$

where:

$$
\mathcal{C}\left(p ; \iota_{1}, \iota_{2}\right):=\frac{p^{p} \sin ^{p}\left(\frac{\pi}{p}\right)}{\pi^{p}(p-1)}\left(\iota_{2}-\iota_{1}\right)^{p+1} .
$$

Proof. From the $P$-convexity of $\left[\mathcal{\omega}^{\prime}(\mu)\right]^{p}$ on $\left(\iota_{1}, \iota_{2}\right)$, we have:

$$
\begin{gathered}
\frac{1}{\iota_{2}-\iota_{1}} \int_{\iota_{1}}^{\iota_{2}}\left[\boldsymbol{\omega}^{\prime}(\mu)\right]^{p} d \mu=\int_{0}^{1}\left[\mathcal{\omega}^{\prime}\left(\delta \iota_{1}+(1-\delta) \iota_{2}\right)\right]^{p} d \delta \leq \int_{0}^{1}\left(\left[\boldsymbol{\omega}^{\prime}\left(\iota_{1}\right)\right]^{p}+\left[\mathcal{\omega}^{\prime}\left(\iota_{2}\right)\right]^{p}\right) d \delta \\
=\left[\mathcal{\omega}^{\prime}\left(\iota_{1}\right)\right]^{p}+\left[\omega^{\prime}\left(\iota_{2}\right)\right]^{p} .
\end{gathered}
$$

Multiplying by $C\left(p ; \iota_{1}, \iota_{2}\right)$ and using Lemma 1 , we obtain the desired inequality (8).

Theorem 6. Let $\iota_{1}<\iota_{2}$ and $\iota_{1}, \iota_{2} \in I^{\circ}$. Suppose that $\omega$ is a differentiable function on $I$, where $\omega$ and $\omega^{\prime}$ are positive and $\omega\left(\iota_{1}\right)=0$. If $\left[\omega^{\prime}(\mu)\right]^{p}$ is a quasi-convex function on $\left(\iota_{1}, \iota_{2}\right)$, then for all $p>1$, we have:

$$
\int_{\iota_{1}}^{\iota_{2}}[\omega(\mu)]^{p} d \mu \leq \mathcal{C}\left(p ; \iota_{1}, \iota_{2}\right) \max \left\{\left[\mathcal{W}^{\prime}\left(\iota_{1}\right)\right]^{p},\left[\mathcal{W}^{\prime}\left(\iota_{2}\right)\right]^{p}\right\},
$$

where $\mathcal{C}\left(p ; \iota_{1}, \iota_{2}\right)$ is defined as in Theorem 5.

Proof. From the quasi-convexity of $\left[\omega^{\prime}(\mu)\right]^{p}$ on $\left(\iota_{1}, \iota_{2}\right)$, we have:

$$
\begin{gathered}
\frac{1}{\iota_{2}-\iota_{1}} \int_{\iota_{1}}^{\iota_{2}}\left[\mathcal{\omega}^{\prime}(\mu)\right]^{p} d \mu=\int_{0}^{1}\left[\mathcal{\omega}^{\prime}\left(\delta \iota_{1}+(1-\delta) \iota_{2}\right)\right]^{p} d \delta \leq \int_{0}^{1} \max \left\{\left[\mathcal{\omega}^{\prime}\left(\iota_{1}\right)\right]^{p},\left[\mathcal{W}^{\prime}\left(\iota_{2}\right)\right]^{p}\right\} d \delta \\
=\max \left\{\left[\mathcal{W}^{\prime}\left(\iota_{1}\right)\right]^{p},\left[\mathcal{\omega}^{\prime}\left(\iota_{2}\right)\right]^{p}\right\} .
\end{gathered}
$$

Multiplying by $\mathcal{C}\left(p ; \iota_{1}, \iota_{2}\right)$ and using Lemma 1 , we obtain the desired inequality (9).

Theorem 7. Let $\iota_{1}<\iota_{2}$ and $\iota_{1}, \iota_{2} \in I^{\circ}$. Assume that $\omega$ is a differentiable function on $I$, where $\omega$ and $\omega^{\prime}$ are positive and $\omega\left(\iota_{1}\right)=0$. If $\left[\omega^{\prime}(\mu)\right]^{p}$ is a convex function on $\left(\iota_{1}, \iota_{2}\right)$, then for all $p>1$, we have:

$$
\int_{\iota_{1}}^{\iota_{2}}[\omega(\mu)]^{p} d \mu \leq \mathcal{C}\left(p ; \iota_{1}, \iota_{2}\right)\left\{\frac{\left[\omega^{\prime}\left(\iota_{1}\right)\right]^{p}+\left[\omega^{\prime}\left(\iota_{2}\right)\right]^{p}}{2}\right\},
$$


where $\mathcal{C}\left(p ; \iota_{1}, \iota_{2}\right)$ is defined as in Theorem 5.

Proof. From the convexity of $\left[\omega^{\prime}(\mu)\right]^{p}$ on $\left(\iota_{1}, \iota_{2}\right)$, we have:

$$
\begin{gathered}
\frac{1}{\iota_{2}-\iota_{1}} \int_{\iota_{1}}^{\iota_{2}}\left[\omega^{\prime}(\mu)\right]^{p} d \mu=\int_{0}^{1}\left[\omega ^ { \prime } \left(\delta \iota_{1}+\right.\right. \\
=\frac{\left[\omega^{\prime}\left(\iota_{1}\right)\right]^{p}+\left[\omega^{\prime}\left(\iota_{2}\right)\right]^{p}}{2} .
\end{gathered}
$$

Multiplying by $\mathcal{C}\left(p ; \iota_{1}, \iota_{2}\right)$ and using Lemma 1 , we obtain the desired inequality $(10)$.

Theorem 8. Let $\iota_{1}<\iota_{2}$ and $\iota_{1}, \iota_{2} \in I^{\circ}$. Suppose that $\omega$ is a differentiable function on $I$, where $\omega$ and $\omega^{\prime}$ are positive and $\omega\left(\iota_{1}\right)=0$. If $\left[\omega^{\prime}(\mu)\right]^{p}$ is an s-convex function on $\left(\iota_{1}, \iota_{2}\right)$, then for all $p>1$, we have:

$$
\int_{\iota_{1}}^{\iota_{2}}[\omega(\mu)]^{p} d \mu \leq \mathcal{C}\left(p ; \iota_{1}, \iota_{2}\right)\left\{\frac{\left[\omega^{\prime}\left(\iota_{1}\right)\right]^{p}+\left[\omega^{\prime}\left(\iota_{2}\right)\right]^{p}}{s+1}\right\},
$$

where $\mathcal{C}\left(p ; \iota_{1}, \iota_{2}\right)$ is defined as in Theorem 5.

Proof. From the s-convexity of $\left[\mathcal{\omega}^{\prime}(\mu)\right]^{p}$ on $\left(\iota_{1}, \iota_{2}\right)$, we have:

$$
\begin{aligned}
\frac{1}{\iota_{2}-\iota_{1}} \int_{\iota_{1}}^{\iota_{2}}\left[\omega^{\prime}(\mu)\right]^{p} d \mu=\int_{0}^{1}\left[\omega ^ { \prime } \left(\delta \iota_{1}+\right.\right. & \left.\left.(1-\delta) \iota_{2}\right)\right]^{p} d \delta \leq \int_{0}^{1}\left(\delta^{S}\left[\omega^{\prime}\left(\iota_{1}\right)\right]^{p}+(1-\delta)^{S}\left[\omega^{\prime}\left(\iota_{2}\right)\right]^{p}\right) d \delta \\
= & \frac{\left[\omega^{\prime}\left(\iota_{1}\right)\right]^{p}+\left[\omega^{\prime}\left(\iota_{2}\right)\right]^{p}}{s+1} .
\end{aligned}
$$

Multiplying by $\mathcal{C}\left(p ; \iota_{1}, \iota_{2}\right)$ and using Lemma 1 , we obtain the desired inequality (11).

Remark 1. Taking $s=1$ in Theorem 8, we obtain Theorem 7.

Theorem 9. Let $\iota_{1}<\iota_{2}$ and $\iota_{1}, \iota_{2} \in I^{\circ}$. Assume that $\omega$ is a differentiable function on $I$, where $\omega$ and $\omega^{\prime}$ are positive, and $\omega\left(\iota_{1}\right)=0$. If $\left[\omega^{\prime}(\mu)\right]^{p}$ is an m-convex function on $\left(\iota_{1}, \iota_{2}\right)$, then for all $p>1$ and $m \in(0,1]$, we have:

$$
\begin{gathered}
\int_{\iota_{1}}^{\iota_{2}}[\omega(\mu)]^{p} d \mu \leq \mathcal{C}\left(p ; \iota_{1}, \iota_{2}\right) \frac{\left(\iota_{1}-m \iota_{2}\right)}{\iota_{2}-\iota_{1}} \\
\times\left\{\frac{1}{2}\left[\left(\frac{\iota_{2}(1-m)}{\iota_{1}-m \iota_{2}}\right)^{2}-1\right]\left[\omega^{\prime}\left(\iota_{1}\right)\right]^{p}+\left[m\left(\frac{\iota_{2}(1-m)}{\iota_{1}-m \iota_{2}}-1\right)-\frac{m}{2}\left(\left(\frac{\iota_{2}(1-m)}{\iota_{1}-m \iota_{2}}\right)^{2}-1\right)\right]\left[\omega^{\prime}\left(\iota_{2}\right)\right]^{p}\right\},
\end{gathered}
$$

where $\mathcal{C}\left(p ; \iota_{1}, \iota_{2}\right)$ is defined as in Theorem 5.

Proof. From the $m$-convexity of $\left[\mathcal{\omega}^{\prime}(\mu)\right]^{p}$ on $\left(\iota_{1}, \iota_{2}\right)$, we have:

$$
\begin{gathered}
\frac{1}{\iota_{2}-\iota_{1}} \int_{\iota_{1}}^{\iota_{2}}\left[\omega^{\prime}(\mu)\right]^{p} d \mu=\frac{\left(\iota_{1}-m \iota_{2}\right)}{\iota_{2}-\iota_{1}} \int_{1}^{\frac{\iota_{2}(1-m)}{\iota_{1}-m \iota_{2}}}\left[\omega^{\prime}\left(\delta \iota_{1}+m(1-\delta) \iota_{2}\right)\right]^{p} d \delta \\
\leq \frac{\left(\iota_{1}-m \iota_{2}\right)}{\iota_{2}-\iota_{1}} \int_{1}^{\frac{\iota_{2}(1-m)}{l_{1}-m \iota_{2}}}\left(\delta\left[\omega^{\prime}\left(\iota_{1}\right)\right]^{p}+m(1-\delta)\left[\omega^{\prime}\left(\iota_{2}\right)\right]^{p}\right) d \delta \\
\times\left\{\frac{1}{2}\left[\left(\frac{\iota_{2}(1-m)}{\iota_{1}-m \iota_{2}}\right)^{2}-1\right]\left[\omega^{\prime}\left(\iota_{1}\right)\right]^{p}+\left[m\left(\frac{\iota_{2}(1-m)}{\iota_{1}-\iota_{1}}-1\right)-\frac{m}{2}\left(\left(\frac{\iota_{2}(1-m)}{\iota_{1}-m \iota_{2}}\right)^{2}-1\right)\right]\left[\omega^{\prime}\left(\iota_{2}\right)\right]^{p}\right\} .
\end{gathered}
$$

Multiplying by $\mathcal{C}\left(p ; \iota_{1}, \iota_{2}\right)$ and using Lemma 1 , we obtain the desired inequality (12).

Remark 2. Taking $m=1$ in Theorem 9, we obtain Theorem 7. 
Theorem 10. Let $\iota_{1}<\iota_{2}$ and $\iota_{1}, \iota_{2} \in I^{\circ}$. Suppose that $\omega$ is a differentiable function on $I$, where $\omega$ and $\omega^{\prime}$ are positive and $\omega\left(\iota_{1}\right)=0$. If $\left[\omega^{\prime}(\mu)\right]^{p}$ is an $(\alpha, m)$-convex function on $\left(\iota_{1}, \iota_{2}\right)$, then for all $p>1$ and $(\alpha, m) \in(0,1]^{2}$, we have:

$$
\begin{gathered}
\int_{\iota_{1}}^{\iota_{2}}[\omega(\mu)]^{p} d \mu \leq \mathcal{C}\left(p ; \iota_{1}, \iota_{2}\right) \frac{\left(\iota_{1}-m \iota_{2}\right)}{\iota_{2}-\iota_{1}} \\
\times\left\{\frac{1}{\alpha+1}\left[\left(\frac{\iota_{2}(1-m)}{\iota_{1}-m \iota_{2}}\right)^{\alpha+1}-1\right]\left[\omega^{\prime}\left(\iota_{1}\right)\right]^{p}\right. \\
\left.+\left[m\left(\frac{\iota_{2}(1-m)}{\iota_{1}-m \iota_{2}}-1\right)-\frac{m}{\alpha+1}\left(\left(\frac{\iota_{2}(1-m)}{\iota_{1}-m \iota_{2}}\right)^{\alpha+1}-1\right)\right]\left[\omega^{\prime}\left(\iota_{2}\right)\right]^{p}\right\},
\end{gathered}
$$

where $\mathcal{C}\left(p ; \iota_{1}, \iota_{2}\right)$ is defined as in Theorem 5.

Proof. From the $(\alpha, m)$-convexity of $\left[\omega^{\prime}(\mu)\right]^{p}$ on $\left(\iota_{1}, \iota_{2}\right)$, we have:

$$
\begin{gathered}
\frac{1}{\iota_{2}-\iota_{1}} \int_{\iota_{1}}^{\iota_{2}}\left[\boldsymbol{\omega}^{\prime}(\mu)\right]^{p} d \mu=\frac{\left(\iota_{1}-m \iota_{2}\right)}{\iota_{2}-\iota_{1}} \int_{1}^{\frac{\iota_{2}(1-m)}{\iota_{1}-m \iota_{2}}}\left[\omega^{\prime}\left(\delta \iota_{1}+m(1-\delta) \iota_{2}\right)\right]^{p} d \delta \\
\leq \frac{\left(\iota_{1}-m \iota_{2}\right)}{\iota_{2}-\iota_{1}} \int_{1}^{\frac{\iota_{2}\left(1-m \iota^{2}\right.}{\iota_{1}-m}}\left(\delta^{\alpha}\left[\boldsymbol{\omega}^{\prime}\left(\iota_{1}\right)\right]^{p}+m\left(1-\delta^{\alpha}\right)\left[\boldsymbol{\omega}^{\prime}\left(\iota_{2}\right)\right]^{p}\right) d \delta \\
=\frac{\left(\iota_{1}-m \iota_{2}\right)}{\iota_{2}-\iota_{1}}\left\{\frac{1}{\alpha+1}\left[\left(\frac{\iota_{2}(1-m)}{\iota_{1}-m \iota_{2}}\right)^{\alpha+1}-1\right]\left[\omega^{\prime}\left(\iota_{1}\right)\right]^{p}\right. \\
\left.+\left[m\left(\frac{\iota_{2}(1-m)}{\iota_{1}-m \iota_{2}}-1\right)-\frac{m}{\alpha+1}\left(\left(\frac{\iota_{2}(1-m)}{\iota_{1}-m \iota_{2}}\right)^{\alpha+1}-1\right)\right]\left[\boldsymbol{\omega}^{\prime}\left(\iota_{2}\right)\right]^{p}\right\} .
\end{gathered}
$$

Multiplying by $\mathcal{C}\left(p ; \iota_{1}, \iota_{2}\right)$ and using Lemma 1 , we obtain the desired inequality (13).

Remark 3. Taking $\alpha=1$ in Theorem 10, we obtain Theorem 9.

Remark 4. Our above results still hold if we apply Lemma 2, so we omit their proofs.

Theorem 11. Let $-\infty<\iota_{1}<\iota_{2}<\infty$ and $\omega$ be an $n$-times differentiable function on $\left(\iota_{1}, \iota_{2}\right)$ such that $\omega, \omega^{\prime}, \ldots, \omega^{(n)}$ are positive with $\omega^{(j)}\left(\iota_{1}\right)=0$, for all $j=0,1, \ldots, n-1$. If $\left[\omega^{(n)}(\mu)\right]^{p}$ is a P-convex function on $\left(\iota_{1}, \iota_{2}\right)$, then for all $p>1$, we have:

$$
\int_{\iota_{1}}^{\iota_{2}}[\omega(\mu)]^{p} d \mu \leq \mathcal{D}\left(n, p ; \iota_{1}, \iota_{2}\right)\left\{\left[\omega^{(n)}\left(\iota_{1}\right)\right]^{p}+\left[\omega^{(n)}\left(\iota_{2}\right)\right]^{p}\right\},
$$

where:

$$
\mathcal{D}\left(n, p ; \iota_{1}, \iota_{2}\right):=\left(\frac{p^{p} \sin ^{p}\left(\frac{\pi}{p}\right)}{\pi^{p}(p-1)}\right)^{n}\left(\iota_{2}-\iota_{1}\right)^{n p+1} .
$$

Proof. From the $P$-convexity of $\left[\omega^{(n)}(\mu)\right]^{p}$ on $\left(\iota_{1}, \iota_{2}\right)$, we have:

$$
\begin{gathered}
\frac{1}{\iota_{2}-\iota_{1}} \int_{\iota_{1}}^{\iota_{2}}\left[\omega^{(n)}(\mu)\right]^{p} d \mu=\int_{0}^{1}\left[\omega^{(n)}\left(\delta \iota_{1}+(1-\delta) \iota_{2}\right)\right]^{p} d \delta \leq \int_{0}^{1}\left(\left[\omega^{(n)}\left(\iota_{1}\right)\right]^{p}+\left[\omega^{(n)}\left(\iota_{2}\right)\right]^{p}\right) d \delta \\
=\left[\omega^{(n)}\left(\iota_{1}\right)\right]^{p}+\left[\omega^{(n)}\left(\iota_{2}\right)\right]^{p} .
\end{gathered}
$$

Multiplying by $\mathcal{D}\left(n, p ; \iota_{1}, \iota_{2}\right)$ and using Lemma 3 , we obtain the desired inequality (14).

Theorem 12. Let $-\infty<\iota_{1}<\iota_{2}<\infty$ and $\omega$ be an $n$-times differentiable function on $\left(\iota_{1}, \iota_{2}\right)$ such that $\omega, \omega^{\prime}, \ldots, \omega^{(n)}$ are positive with $\omega^{(j)}\left(\iota_{1}\right)=0$, for all $j=0,1, \ldots, n-1$. If $\left[\omega^{(n)}(\mu)\right]^{p}$ is a quasi-convex function on $\left(\iota_{1}, \iota_{2}\right)$, then for all $p>1$, we have: 


$$
\int_{\iota_{1}}^{\iota_{2}}[\mathcal{\omega}(\mu)]^{p} d \mu \leq \mathcal{D}\left(n, p ; \iota_{1}, \iota_{2}\right) \max \left\{\left[\mathcal{W}^{(n)}\left(\iota_{1}\right)\right]^{p},\left[\mathcal{W}^{(n)}\left(\iota_{2}\right)\right]^{p}\right\},
$$

where $\mathcal{D}\left(n, p ; \iota_{1}, \iota_{2}\right)$ is defined as in Theorem 11 .

Proof. From the quasi-convexity of $\left[\mathcal{\omega}^{(n)}(\mu)\right]^{p}$ on $\left(\iota_{1}, \iota_{2}\right)$, we have:

$$
\begin{gathered}
\frac{1}{\iota_{2}-\iota_{1}} \int_{\iota_{1}}^{\iota_{2}}\left[\mathfrak{\omega}^{(n)}(\mu)\right]^{p} d \mu=\int_{0}^{1}\left[\mathfrak{\omega}^{(n)}\left(\delta \iota_{1}+(1-\delta) \iota_{2}\right)\right]^{p} d \delta \leq \int_{0}^{1} \max \left\{\left[\mathfrak{\omega}^{(n)}\left(\iota_{1}\right)\right]^{p},\left[\mathfrak{\omega}^{(n)}\left(\iota_{2}\right)\right]^{p}\right\} d \delta \\
=\max \left\{\left[\mathfrak{\omega}^{(n)}\left(\iota_{1}\right)\right]^{p},\left[\mathfrak{\omega}^{(n)}\left(\iota_{2}\right)\right]^{p}\right\} .
\end{gathered}
$$

Multiplying by $\mathcal{D}\left(n, p ; \iota_{1}, \iota_{2}\right)$ and using Lemma 3 , we obtain the desired inequality (15).

Theorem 13. Let $-\infty<\iota_{1}<\iota_{2}<\infty$ and $\omega$ be an $n$-times differentiable function on $\left(\iota_{1}, \iota_{2}\right)$ such that $\omega, \omega^{\prime}, \ldots, \omega^{(n)}$ are positive with $\omega^{(j)}\left(\iota_{1}\right)=0$, for all $j=0,1, \ldots, n-1$. If $\left[\omega^{(n)}(\mu)\right]^{p}$ is a convex function on $\left(\iota_{1}, \iota_{2}\right)$, then for all $p>1$, we have:

$$
\int_{\iota_{1}}^{\iota_{2}}[\omega(\mu)]^{p} d \mu \leq \mathcal{D}\left(n, p ; \iota_{1}, \iota_{2}\right)\left\{\frac{\left[\omega^{(n)}\left(\iota_{1}\right)\right]^{p}+\left[\omega^{(n)}\left(\iota_{2}\right)\right]^{p}}{2}\right\},
$$

where $D\left(n, p ; \iota_{1}, \iota_{2}\right)$ is defined as in Theorem 11 .

Proof. From the convexity of $\left[\omega^{(n)}(\mu)\right]^{p}$ on $\left(\iota_{1}, \iota_{2}\right)$, we have:

$$
\begin{gathered}
\frac{1}{\iota_{2}-\iota_{1}} \int_{\iota_{1}}^{\iota_{2}}\left[\mathcal{\omega}^{(n)}(\mu)\right]^{p} d \mu=\int_{0}^{1}\left[\omega^{(n)}\left(\delta \iota_{1}+(1-\delta) \iota_{2}\right)\right]^{p} d \delta \\
\leq \int_{0}^{1}\left(\delta\left[\omega^{(n)}\left(\iota_{1}\right)\right]^{p}+(1-\delta)\left[\omega^{(n)}\left(\iota_{2}\right)\right]^{p}\right) d \delta \\
=\frac{\left[\omega^{(n)}\left(\iota_{1}\right)\right]^{p}+\left[\omega^{(n)}\left(\iota_{2}\right)\right]^{p}}{2} .
\end{gathered}
$$

Multiplying by $\mathcal{D}\left(n, p ; \iota_{1}, \iota_{2}\right)$ and using Lemma 3 , we obtain the desired inequality (16).

Theorem 14. Let $-\infty<\iota_{1}<\iota_{2}<\infty$ and $\omega$ be an $n$-times differentiable function on $\left(\iota_{1}, \iota_{2}\right)$ such that $\omega, \omega^{\prime}, \ldots, \omega^{(n)}$ are positive with $\omega^{(j)}\left(\iota_{1}\right)=0$, for all $j=0,1, \ldots, n-1$. If $\left[\omega^{(n)}(\mu)\right]^{p}$ is an s-convex function on $\left(\iota_{1}, \iota_{2}\right)$, then for all $p>1$, we have:

$$
\int_{\iota_{1}}^{\iota_{2}}[\omega(\mu)]^{p} d \mu \leq \mathcal{D}\left(n, p ; \iota_{1}, \iota_{2}\right)\left\{\frac{\left[\omega^{(n)}\left(\iota_{1}\right)\right]^{p}+\left[\omega^{(n)}\left(\iota_{2}\right)\right]^{p}}{s+1}\right\},
$$

where $\mathcal{D}\left(n, p ; l_{1}, \iota_{2}\right)$ is defined as in Theorem 11.

Proof. From the s-convexity of $\left[\omega^{(n)}(\mu)\right]^{p}$ on $\left(\iota_{1}, \iota_{2}\right)$, we have:

$$
\begin{gathered}
\frac{1}{\iota_{2}-\iota_{1}} \int_{\iota_{1}}^{\iota_{2}}\left[\omega^{(n)}(\mu)\right]^{p} d \mu=\int_{0}^{1}\left[\omega^{(n)}\left(\delta \iota_{1}+(1-\delta) \iota_{2}\right)\right]^{p} d \delta \\
\leq \int_{0}^{1}\left(\delta^{s}\left[\omega^{(n)}\left(\iota_{1}\right)\right]^{p}+(1-\delta)^{s}\left[\omega^{(n)}\left(\iota_{2}\right)\right]^{p}\right) d \delta \\
=\frac{\left[\omega^{(n)}\left(\iota_{1}\right)\right]^{p}+\left[\omega^{(n)}\left(\iota_{2}\right)\right]^{p}}{s+1} .
\end{gathered}
$$

Multiplying by $\mathcal{D}\left(n, p ; \iota_{1}, \iota_{2}\right)$ and using Lemma 3 , we obtain the desired inequality (17).

Remark 5. Taking $s=1$ in Theorem 14, we obtain Theorem 13. 
Theorem 15. Let $-\infty<\iota_{1}<\iota_{2}<\infty$ and $\omega$ be an $n$-times differentiable function on $\left(\iota_{1}, \iota_{2}\right)$ such that $\omega, \omega^{\prime}, \ldots, \omega^{(n)}$ are positive with $\omega^{(j)}\left(\iota_{1}\right)=0$, for all $j=0,1, \ldots, n-1$. If $\left[\omega^{(n)}(\mu)\right]^{p}$ is an $m$-convex function on $\left(\iota_{1}, \iota_{2}\right)$, then for all $p>1$ and $m \in(0,1]$, we have:

$$
\begin{gathered}
\int_{\iota_{1}}^{\iota_{2}}[\omega(\mu)]^{p} d \mu \leq \mathcal{D}\left(n, p ; \iota_{1}, \iota_{2}\right) \frac{\left(\iota_{1}-m \iota_{2}\right)}{\iota_{2}-\iota_{1}} \\
\times\left\{\frac{1}{2}\left[\left(\frac{\iota_{2}(1-m)}{\iota_{1}-m \iota_{2}}\right)^{2}-1\right]\left[\omega^{(n)}\left(\iota_{1}\right)\right]^{p}\right. \\
\left.+\left[m\left(\frac{\iota_{2}(1-m)}{\iota_{1}-m \iota_{2}}-1\right)-\frac{m}{2}\left(\left(\frac{\iota_{2}(1-m)}{\iota_{1}-m \iota_{2}}\right)^{2}-1\right)\right]\left[\omega^{(n)}\left(\iota_{2}\right)\right]^{p}\right\},
\end{gathered}
$$

where $\mathcal{D}\left(n, p ; \iota_{1}, \iota_{2}\right)$ is defined as in Theorem 11.

Proof. From the $m$-convexity of $\left[\omega^{(n)}(\mu)\right]^{p}$ on $\left(\iota_{1}, \iota_{2}\right)$, we have:

$$
\begin{gathered}
\frac{1}{\iota_{2}-\iota_{1}} \int_{\iota_{1}}^{\iota_{2}}\left[\omega^{(n)}(\mu)\right]^{p} d \mu=\frac{\left(\iota_{1}-m \iota_{2}\right)}{\iota_{2}-\iota_{1}} \int_{1}^{\frac{\iota_{2}(1-m)}{\iota_{1}-m \iota_{2}}}\left[\omega^{(n)}\left(\delta \iota_{1}+m(1-\delta) \iota_{2}\right)\right]^{p} d \delta \\
\leq \frac{\left(\iota_{1}-m \iota_{2}\right)}{\iota_{2}-\iota_{1}} \int_{1}^{\frac{\iota-m \iota_{2}}{\iota_{1}-m}}\left(\delta\left[\omega^{(n)}\left(\iota_{1}\right)\right]^{p}+m(1-\delta)\left[\omega^{(n)}\left(\iota_{2}\right)\right]^{p}\right) d \delta \\
=\frac{\left(\iota_{1}-m \iota_{2}\right)}{\iota_{2}-\iota_{1}}\left\{\frac{1}{2}\left[\left(\frac{\iota_{2}(1-m)}{\iota_{1}-m \iota_{2}}\right)^{2}-1\right]\left[\omega^{(n)}\left(\iota_{1}\right)\right]^{p}\right. \\
\left.+\left[m\left(\frac{\iota_{2}(1-m)}{\iota_{1}-m \iota_{2}}-1\right)-\frac{m}{2}\left(\left(\frac{\iota_{2}(1-m)}{\iota_{1}-m \iota_{2}}\right)^{2}-1\right)\right]\left[\omega^{(n)}\left(\iota_{2}\right)\right]^{p}\right\} .
\end{gathered}
$$

Multiplying by $\mathcal{D}\left(n, p ; \iota_{1}, \iota_{2}\right)$ and using Lemma 3, we obtain the desired inequality (18).

Remark 6. Taking $m=1$ in Theorem 15, we obtain Theorem 13.

Theorem 16. Let $-\infty<\iota_{1}<\iota_{2}<\infty$ and $\omega$ be an $n$-times differentiable function on $\left(\iota_{1}, \iota_{2}\right)$ such that $\omega, \omega^{\prime}, \ldots, \omega^{(n)}$ are positive with $\omega^{(j)}\left(\iota_{1}\right)=0$, for all $j=0,1, \ldots, n-1$. If $\left[\omega^{(n)}(\mu)\right]^{p}$ is an $(\alpha, m)$-convex function on $\left(\iota_{1}, \iota_{2}\right)$, then for all $p>1$ and $(\alpha, m) \in(0,1]^{2}$, we have:

$$
\begin{gathered}
\int_{\iota_{1}}^{\iota_{2}}[\omega(\mu)]^{p} d \mu \leq \mathcal{D}\left(n, p ; \iota_{1}, \iota_{2}\right) \frac{\left(\iota_{1}-m \iota_{2}\right)}{\iota_{2}-\iota_{1}} \\
\times\left\{\frac{1}{\alpha+1}\left[\left(\frac{\iota_{2}(1-m)}{\iota_{1}-m \iota_{2}}\right)^{\alpha+1}-1\right]\left[\omega^{(n)}\left(\iota_{1}\right)\right]^{p}\right. \\
\left.+\left[m\left(\frac{\iota_{2}(1-m)}{\iota_{1}-m \iota_{2}}-1\right)-\frac{m}{\alpha+1}\left(\left(\frac{\iota_{2}(1-m)}{\iota_{1}-m \iota_{2}}\right)^{\alpha+1}-1\right)\right]\left[\omega^{(n)}\left(\iota_{2}\right)\right]^{p}\right\},
\end{gathered}
$$

where $\mathcal{D}\left(n, p ; \iota_{1}, \iota_{2}\right)$ is defined as in Theorem 11.

Proof. From the $(\alpha, m)$-convexity of $\left[\mathcal{\omega}^{(n)}(\mu)\right]^{p}$ on $\left(\iota_{1}, \iota_{2}\right)$, we have:

$$
\begin{gathered}
\frac{1}{\iota_{2}-\iota_{1}} \int_{\iota_{1}}^{\iota_{2}}\left[\mathcal{\omega}^{(n)}(\mu)\right]^{p} d \mu=\frac{\left(\iota_{1}-m \iota_{2}\right)}{\iota_{2}-\iota_{1}} \int_{1}^{\frac{\iota_{2}(1-m)}{\iota_{1}-m \iota_{2}}}\left[\mathcal{\omega}^{(n)}\left(\delta \iota_{1}+m(1-\delta) \iota_{2}\right)\right]^{p} d \delta \\
\leq \frac{\left(\iota_{1}-m \iota_{2}\right)}{\iota_{2}-\iota_{1}} \int_{1}^{\frac{\iota_{2}(1-m)}{\iota_{1}-m \iota_{2}}}\left(\delta^{\alpha}\left[\mathcal{\omega}^{(n)}\left(\iota_{1}\right)\right]^{p}+m\left(1-\delta^{\alpha}\right)\left[\mathcal{\omega}^{(n)}\left(\iota_{2}\right)\right]^{p}\right) d \delta \\
=\frac{\left(\iota_{1}-m \iota_{2}\right)}{\iota_{2}-\iota_{1}}\left\{\frac{1}{\alpha+1}\left[\left(\frac{\iota_{2}(1-m)}{\iota_{1}-m \iota_{2}}\right)^{\alpha+1}-1\right]\left[\mathcal{\omega}^{(n)}\left(\iota_{1}\right)\right]^{p}\right. \\
\left.+\left[m\left(\frac{\iota_{2}(1-m)}{\iota_{1}-m \iota_{2}}-1\right)-\frac{m}{\alpha+1}\left(\left(\frac{\iota_{2}(1-m)}{\iota_{1}-m \iota_{2}}\right)^{\alpha+1}-1\right)\right]\left[\mathcal{\omega}^{(n)}\left(\iota_{2}\right)\right]^{p}\right\} .
\end{gathered}
$$


Multiplying by $\mathcal{D}\left(n, p ; \iota_{1}, \iota_{2}\right)$ and using Lemma 3, we obtain the desired inequality (19).

Remark 7. Taking $m=1$ in Theorem 16, we obtain Theorem 15.

Remark 8. Our above results still holds if we apply Lemma 4, so we omit their proofs.

\section{Inequalities of Ostrowski Type}

The Ostrowski inequality [30] is remarkable and has the following representation:

Theorem 17. Let $\omega: I \rightarrow \mathbb{R}$ be a differentiable function on $I^{\circ}$, with $\iota_{1}, \iota_{2} \in I^{\circ}$ and $\iota_{1}<\iota_{2}$. If $\left|\omega^{\prime}(\mu)\right| \leq M$ for all $\mu \in\left[\iota_{1}, \iota_{2}\right]$, then:

$$
\left|\omega(\mu)-\frac{1}{\iota_{2}-\iota_{1}} \int_{\iota_{1}}^{\iota_{2}} \omega(\delta) d \delta\right| \leq M\left(\iota_{2}-\iota_{1}\right)\left[\frac{1}{4}+\frac{\left(\mu-\frac{\iota_{1}+\iota_{2}}{2}\right)^{2}}{\left(\iota_{2}-\iota_{1}\right)^{2}}\right], \quad \forall \mu \in\left[\iota_{1}, \iota_{2}\right] .
$$

For other recent results of this type, please see $[9,30,31]$ and the references therein.

Theorem 18. Let $\xi \in\left(\iota_{1}, \iota_{2}\right)$ and $\omega$ be a differentiable function on $\left(\iota_{1}, \iota_{2}\right)$, where $\omega$ and $\omega^{\prime}$ are positive with $\omega\left(\iota_{1}\right)=\omega\left(\iota_{2}\right)=0$. If $\left[\omega^{\prime}(\mu)\right]^{p}$ is a P-convex function on $\left(\iota_{1}, \iota_{2}\right)$, then for all $p>1$, we have:

$$
\int_{\iota_{1}}^{\iota_{2}}|\omega(\mu)-\omega(\xi)|^{p} d \mu \leq \mathcal{E}\left(\xi ; p, \iota_{1}, \iota_{2}\right)\left\{\left[\omega^{\prime}\left(\iota_{1}\right)\right]^{p}+\left[\omega^{\prime}\left(\iota_{2}\right)\right]^{p}\right\},
$$

where:

$$
\mathcal{E}\left(\xi ; p, \iota_{1}, \iota_{2}\right):=\frac{p^{p} \sin ^{p}\left(\frac{\pi}{p}\right)}{\pi^{p}(p-1)}\left[\frac{\iota_{2}-\iota_{1}}{2}+\left|\xi-\frac{\iota_{1}+\iota_{2}}{2}\right|\right]^{p}\left(\iota_{2}-\iota_{1}\right) .
$$

Proof. From the $P$-convexity of $\left[\omega^{\prime}(\mu)\right]^{p}$ on $\left(\iota_{1}, \iota_{2}\right)$, we have:

$$
\begin{gathered}
\frac{1}{\iota_{2}-\iota_{1}} \int_{\iota_{1}}^{\iota_{2}}\left[\boldsymbol{\omega}^{\prime}(\mu)\right]^{p} d \mu=\int_{0}^{1}\left[\boldsymbol{\omega}^{\prime}\left(\delta \iota_{1}+(1-\delta) \iota_{2}\right)\right]^{p} d \delta \leq \int_{0}^{1}\left(\left[\boldsymbol{\omega}^{\prime}\left(\iota_{1}\right)\right]^{p}+\left[\boldsymbol{\omega}^{\prime}\left(\iota_{2}\right)\right]^{p}\right) d \delta \\
=\left[\boldsymbol{\omega}^{\prime}\left(\iota_{1}\right)\right]^{p}+\left[\boldsymbol{\omega}^{\prime}\left(\iota_{2}\right)\right]^{p} .
\end{gathered}
$$

Multiplying by $E\left(\xi ; p, \iota_{1}, \iota_{2}\right)$ and using Theorem 3 , we obtain the desired inequality (21).

Theorem 19. Let $\xi \in\left(\iota_{1}, \iota_{2}\right)$ and $\omega$ be a differentiable function on $\left(\iota_{1}, \iota_{2}\right)$, where $\omega$ and $\omega^{\prime}$ are positive with $\omega\left(\iota_{1}\right)=\omega\left(\iota_{2}\right)=0$. If $\left[\omega^{\prime}(\mu)\right]^{p}$ is a quasi-convex function on $\left(\iota_{1}, \iota_{2}\right)$, then for all $p>1$, we have:

$$
\int_{\iota_{1}}^{\iota_{2}}|\omega(\mu)-\omega(\xi)|^{p} d \mu \leq \mathcal{E}\left(\xi ; p, \iota_{1}, \iota_{2}\right) \max \left\{\left[\omega^{\prime}\left(\iota_{1}\right)\right]^{p},\left[\omega^{\prime}\left(\iota_{2}\right)\right]^{p}\right\}
$$

where $\mathcal{E}\left(\xi ; p, \iota_{1}, \iota_{2}\right)$ is defined as in Theorem 18.

Proof. From the quasi-convexity of $\left[\omega^{\prime}(\mu)\right]^{p}$ on $\left(\iota_{1}, \iota_{2}\right)$, we have:

$$
\begin{gathered}
\frac{1}{\iota_{2}-\iota_{1}} \int_{\iota_{1}}^{\iota_{2}}\left[\boldsymbol{\omega}^{\prime}(\mu)\right]^{p} d \mu=\int_{0}^{1}\left[\boldsymbol{\omega}^{\prime}\left(\delta \iota_{1}+(1-\delta) \iota_{2}\right)\right]^{p} d \delta \leq \int_{0}^{1} \max \left\{\left[\omega^{\prime}\left(\iota_{1}\right)\right]^{p},\left[\omega^{\prime}\left(\iota_{2}\right)\right]^{p}\right\} d \delta \\
=\max \left\{\left[\boldsymbol{\omega}^{\prime}\left(\iota_{1}\right)\right]^{p},\left[\boldsymbol{\omega}^{\prime}\left(\iota_{2}\right)\right]^{p}\right\} .
\end{gathered}
$$

Multiplying by $\mathcal{E}\left(\xi ; p, \iota_{1}, \iota_{2}\right)$ and using Theorem 3 , we obtain the desired inequality (22).

Theorem 20. Let $\xi \in\left(\iota_{1}, \iota_{2}\right)$ and $\omega$ be a differentiable function on $\left(\iota_{1}, \iota_{2}\right)$, where $\omega$ and $\omega^{\prime}$ are positive with $\omega\left(\iota_{1}\right)=\omega\left(\iota_{2}\right)=0$. If $\left[\omega^{\prime}(\mu)\right]^{p}$ is a convex function on $\left(\iota_{1}, \iota_{2}\right)$, then for all $p>1$, we have:

$$
\int_{\iota_{1}}^{\iota_{2}}|\omega(\mu)-\omega(\xi)|^{p} d \mu \leq \mathcal{E}\left(\xi ; p, \iota_{1}, \iota_{2}\right)\left\{\frac{\left[\omega^{\prime}\left(\iota_{1}\right)\right]^{p}+\left[\omega^{\prime}\left(\iota_{2}\right)\right]^{p}}{2}\right\},
$$


where $\mathcal{E}\left(\xi ; p, \iota_{1}, \iota_{2}\right)$ is defined as in Theorem 18.

Proof. From the convexity of $\left[\omega^{\prime}(\mu)\right]^{p}$ on $\left(\iota_{1}, \iota_{2}\right)$, we have:

$$
\begin{gathered}
\frac{1}{\iota_{2}-\iota_{1}} \int_{\iota_{1}}^{\iota_{2}}\left[\boldsymbol{\omega}^{\prime}(\mu)\right]^{p} d \mu=\int_{0}^{1}\left[\omega ^ { \prime } \left(\delta \iota_{1}+\right.\right. \\
\left.=\frac{\left.\left.[1-\delta) \iota_{2}\right)\right]^{p} d \delta \leq \int_{0}^{1}\left(\delta\left[\boldsymbol{\omega}^{\prime}\left(\iota_{1}\right)\right]^{p}+\left[\omega^{\prime}\left(\iota_{2}\right)\right]^{p}\right.}{2} .(1-\delta)\left[\boldsymbol{\omega}^{\prime}\left(\iota_{2}\right)\right]^{p}\right) d \delta \\
=
\end{gathered}
$$

Multiplying by $\mathcal{E}\left(\xi ; p, \iota_{1}, \iota_{2}\right)$ and using Theorem 3 , we obtain the desired inequality (23).

Theorem 21. Let $\xi \in\left(\iota_{1}, \iota_{2}\right)$ and $\omega$ be a differentiable function on $\left(\iota_{1}, \iota_{2}\right)$, where $\omega$ and $\omega^{\prime}$ are positive with $\omega\left(\iota_{1}\right)=\omega\left(\iota_{2}\right)=0$. If $\left[\omega^{\prime}(\mu)\right]^{p}$ is an s-convex function on $\left(\iota_{1}, \iota_{2}\right)$, then for all $p>1$, we have:

$$
\int_{\iota_{1}}^{\iota_{2}}|\omega(\mu)-\omega(\xi)|^{p} d \mu \leq \mathcal{E}\left(\xi ; p, \iota_{1}, \iota_{2}\right)\left\{\frac{\left[\omega^{\prime}\left(\iota_{1}\right)\right]^{p}+\left[\omega^{\prime}\left(\iota_{2}\right)\right]^{p}}{s+1}\right\},
$$

where $\mathcal{E}\left(\xi ; p, \iota_{1}, \iota_{2}\right)$ is defined as in Theorem 18.

Proof. From the s-convexity of $\left[\omega^{\prime}(\mu)\right]^{p}$ on $\left(\iota_{1}, \iota_{2}\right)$, we have:

$$
\begin{aligned}
& \frac{1}{\iota_{2}-\iota_{1}} \int_{\iota_{1}}^{\iota_{2}}\left[\omega^{\prime}(\mu)\right]^{p} d \mu=\int_{0}^{1}\left[\omega^{\prime}\left(\delta \iota_{1}+(1-\delta) \iota_{2}\right)\right]^{p} d \delta \leq \int_{0}^{1}\left(\delta^{S}\left[\omega^{\prime}\left(\iota_{1}\right)\right]^{p}+(1-\delta)^{S}\left[\omega^{\prime}\left(\iota_{2}\right)\right]^{p}\right) d \delta \\
& =\frac{\left[\omega^{\prime}\left(\iota_{1}\right)\right]^{p}+\left[\omega^{\prime}\left(\iota_{2}\right)\right]^{p}}{S+1} \text {. }
\end{aligned}
$$

Multiplying by $\mathcal{E}\left(\xi ; p, \iota_{1}, \iota_{2}\right)$ and using Theorem 3 , we obtain the desired inequality (24).

Remark 9. Taking $s=1$ in Theorem 21, we obtain Theorem 20.

Theorem 22. Let $\xi \in\left(\iota_{1}, \iota_{2}\right)$ and $\omega$ be a differentiable function on $\left(\iota_{1}, \iota_{2}\right)$, where $\omega$ and $\omega^{\prime}$ are positive with $\omega\left(\iota_{1}\right)=\omega\left(\iota_{2}\right)=0$. If $\left[\omega^{\prime}(\mu)\right]^{p}$ is an m-convex function on $\left(\iota_{1}, \iota_{2}\right)$, then for all $p>1$ and $m \in(0,1]$, we have:

$$
\begin{gathered}
\int_{\iota_{1}}^{\iota_{2}}|\omega(\mu)-\omega(\xi)|^{p} d \mu \leq \mathcal{E}\left(\xi ; p, \iota_{1}, \iota_{2}\right) \frac{\left(\iota_{1}-m \iota_{2}\right)}{\iota_{2}-\iota_{1}} \\
\times\left\{\frac{1}{2}\left[\left(\frac{\iota_{2}(1-m)}{\iota_{1}-m \iota_{2}}\right)^{2}-1\right]\left[\omega^{\prime}\left(\iota_{1}\right)\right]^{p}+\left[m\left(\frac{\iota_{2}(1-m)}{\iota_{1}-m \iota_{2}}-1\right)-\frac{m}{2}\left(\left(\frac{\iota_{2}(1-m)}{\iota_{1}-m \iota_{2}}\right)^{2}-1\right)\right]\left[\omega^{\prime}\left(\iota_{2}\right)\right]^{p}\right\},
\end{gathered}
$$

where $\mathcal{E}\left(\xi ; p, \iota_{1}, \iota_{2}\right)$ is defined as in Theorem 18 .

Proof. From the $m$-convexity of $\left[\omega^{\prime}(\mu)\right]^{p}$ on $\left(\iota_{1}, \iota_{2}\right)$, we have:

$$
\begin{gathered}
\frac{1}{\iota_{2}-\iota_{1}} \int_{\iota_{1}}^{\iota_{2}}\left[\omega^{\prime}(\mu)\right]^{p} d \mu=\frac{\left(\iota_{1}-m \iota_{2}\right)}{\iota_{2}-\iota_{1}} \int_{1}^{\frac{\iota_{2}(1-m)}{\iota_{1}-m \iota_{2}}}\left[\omega^{\prime}\left(\delta \iota_{1}+m(1-\delta) \iota_{2}\right)\right]^{p} d \delta \\
\leq \frac{\left(\iota_{1}-m \iota_{2}\right)}{\iota_{2}-\iota_{1}} \int_{1}^{\frac{\iota_{2}(1-m)}{\iota_{1}-m \iota_{2}}}\left(\delta\left[\omega^{\prime}\left(\iota_{1}\right)\right]^{p}+m(1-\delta)\left[\omega^{\prime}\left(\iota_{2}\right)\right]^{p}\right) d \delta \\
\times\left\{\frac{1}{2}\left[\left(\frac{\iota_{2}(1-m)}{\iota_{1}-m \iota_{2}}\right)^{2}-1\right]\left[\omega^{\prime}\left(\iota_{1}\right)\right]^{p}+\left[m\left(\frac{\iota_{2}(1-m)}{\iota_{1}-m \iota_{2}}-1\right)-\frac{m}{2}\left(\left(\frac{\iota_{2}(1-m)}{\iota_{1}-m \iota_{2}}\right)^{2}-1\right)\right]\left[\omega^{\prime}\left(\iota_{2}\right)\right]^{p}\right\} .
\end{gathered}
$$

Multiplying by $\mathcal{E}\left(\xi ; p, \iota_{1}, \iota_{2}\right)$ and using Theorem 3 , we obtain the desired inequality (25).

Remark 10. Taking $m=1$ in Theorem 22, we obtain Theorem 20. 
Theorem 23. Let $\xi \in\left(\iota_{1}, \iota_{2}\right)$ and $\omega$ be a differentiable function on $\left(\iota_{1}, \iota_{2}\right)$, where $\omega$ and $\omega^{\prime}$ are positive with $\omega\left(\iota_{1}\right)=\omega\left(\iota_{2}\right)=0$. If $\left[\omega^{\prime}(\mu)\right]^{p}$ is an $(\alpha, m)$-convex function on $\left(\iota_{1}, \iota_{2}\right)$, then for all $p>1$ and $(\alpha, m) \in(0,1]^{2}$, we have:

$$
\begin{gathered}
\int_{\iota_{1}}^{\iota_{2}}|\omega(\mu)-\omega(\xi)|^{p} d \mu \leq \mathcal{E}\left(\xi ; p, \iota_{1}, \iota_{2}\right) \frac{\left(\iota_{1}-m \iota_{2}\right)}{\iota_{2}-\iota_{1}} \\
\times\left\{\frac{1}{\alpha+1}\left[\left(\frac{\iota_{2}(1-m)}{\iota_{1}-m \iota_{2}}\right)^{\alpha+1}-1\right]\left[\mathcal{\omega}^{\prime}\left(\iota_{1}\right)\right]^{p}\right. \\
\left.+\left[m\left(\frac{\iota_{2}(1-m)}{\iota_{1}-m \iota_{2}}-1\right)-\frac{m}{\alpha+1}\left(\left(\frac{\iota_{2}(1-m)}{\iota_{1}-m \iota_{2}}\right)^{\alpha+1}-1\right)\right]\left[\omega^{\prime}\left(\iota_{2}\right)\right]^{p}\right\},
\end{gathered}
$$

where $\mathcal{E}\left(\xi ; p, \iota_{1}, \iota_{2}\right)$ is defined as in Theorem 18.

Proof. From the $(\alpha, m)$-convexity of $\left[\omega^{\prime}(\mu)\right]^{p}$ on $\left(\iota_{1}, \iota_{2}\right)$, we have:

$$
\begin{gathered}
\frac{1}{\iota_{2}-\iota_{1}} \int_{\iota_{1}}^{\iota_{2}}\left[\omega^{\prime}(\mu)\right]^{p} d \mu=\frac{\left(\iota_{1}-m \iota_{2}\right)}{\iota_{2}-\iota_{1}} \int_{1}^{\frac{\iota_{2}(1-m)}{\iota_{1}-m \iota_{2}}}\left[\omega^{\prime}\left(\delta \iota_{1}+m(1-\delta) \iota_{2}\right)\right]^{p} d \delta \\
\leq \frac{\left(\iota_{1}-m \iota_{2}\right)}{\iota_{2}-\iota_{1}} \int_{1}^{\frac{\iota_{2}\left(1-m \iota^{2}\right.}{\iota_{1}-m}}\left(\delta^{\alpha}\left[\omega^{\prime}\left(\iota_{1}\right)\right]^{p}+m\left(1-\delta^{\alpha}\right)\left[\omega^{\prime}\left(\iota_{2}\right)\right]^{p}\right) d \delta \\
=\frac{\left(\iota_{1}-m \iota_{2}\right)}{\iota_{2}-\iota_{1}}\left\{\frac{1}{\alpha+1}\left[\left(\frac{\iota_{2}(1-m)}{\iota_{1}-m \iota_{2}}\right)^{\alpha+1}-1\right]\left[\omega^{\prime}\left(\iota_{1}\right)\right]^{p}\right. \\
\left.+\left[m\left(\frac{\iota_{2}(1-m)}{\iota_{1}-m \iota_{2}}-1\right)-\frac{m}{\alpha+1}\left(\left(\frac{\iota_{2}(1-m)}{\iota_{1}-m \iota_{2}}\right)^{\alpha+1}-1\right)\right]\left[\omega^{\prime}\left(\iota_{2}\right)\right]^{p}\right\} .
\end{gathered}
$$

Multiplying by $\mathcal{E}\left(\xi ; p, \iota_{1}, \iota_{2}\right)$ and using Theorem 3 , we obtain the desired inequality (26).

Remark 11. Taking $\alpha=1$ in Theorem 23, we obtain Theorem 22.

Theorem 24. Let $\xi \in\left(\iota_{1}, \iota_{2}\right)$ and $\omega$ be an $n$-times differentiable function on $\left(\iota_{1}, \iota_{2}\right)$, where $\omega, \omega^{\prime}, \ldots, \omega^{(n)}$ are positive with $\omega^{(j)}\left(\iota_{1}\right)=\omega^{(j)}\left(\iota_{2}\right)=0$, for all $j=0,1, \ldots, n-1$. If $\left[\omega^{(n)}(\mu)\right]^{p}$ is a P-convex function on $\left(\iota_{1}, \iota_{2}\right)$, then for all $p>1$, we have:

$$
\int_{\iota_{1}}^{\iota_{2}}|\omega(\mu)-\omega(\xi)|^{p} d \mu \leq \mathcal{F}\left(\xi ; n, p, \iota_{1}, \iota_{2}\right)\left\{\left[\omega^{(n)}\left(\iota_{1}\right)\right]^{p}+\left[\omega^{(n)}\left(\iota_{2}\right)\right]^{p}\right\},
$$

where:

$$
\mathcal{F}\left(\xi ; n, p, \iota_{1}, \iota_{2}\right):=\left(\frac{p^{p} \sin ^{p}\left(\frac{\pi}{p}\right)}{\pi^{p}(p-1)}\right)^{n}\left[\frac{\iota_{2}-\iota_{1}}{2}+\left|\xi-\frac{\iota_{1}+\iota_{2}}{2}\right|\right]^{n p}\left(\iota_{2}-\iota_{1}\right) .
$$

Proof. From the $P$-convexity of $\left[\omega^{(n)}(\mu)\right]^{p}$ on $\left(\iota_{1}, \iota_{2}\right)$, we have:

$$
\begin{gathered}
\frac{1}{\iota_{2}-\iota_{1}} \int_{\iota_{1}}^{\iota_{2}}\left[\omega^{(n)}(\mu)\right]^{p} d \mu=\int_{0}^{1}\left[\omega^{(n)}\left(\delta \iota_{1}+(1-\delta) \iota_{2}\right)\right]^{p} d \delta \leq \int_{0}^{1}\left(\left[\omega^{(n)}\left(\iota_{1}\right)\right]^{p}+\left[\omega^{(n)}\left(\iota_{2}\right)\right]^{p}\right) d \delta \\
=\left[\omega^{(n)}\left(\iota_{1}\right)\right]^{p}+\left[\omega^{(n)}\left(\iota_{2}\right)\right]^{p} .
\end{gathered}
$$

Multiplying by $\mathcal{F}\left(\xi ; n, p, \iota_{1}, \iota_{2}\right)$ and using Theorem 4 , we obtain the desired inequality (27). 
Theorem 25. Let $\xi \in\left(\iota_{1}, \iota_{2}\right)$ and $\omega$ be an $n$-times differentiable function on $\left(\iota_{1}, \iota_{2}\right)$, where $\omega, \omega^{\prime}, \ldots, \omega^{(n)}$ are positive with $\omega^{(j)}\left(\iota_{1}\right)=\omega^{(j)}\left(\iota_{2}\right)=0$, for all $j=0,1, \ldots, n-1$. If $\left[\omega^{(n)}(\mu)\right]^{p}$ is a quasi-convex function on $\left(\iota_{1}, \iota_{2}\right)$, then for all $p>1$, we have:

$$
\int_{\iota_{1}}^{\iota_{2}}|\omega(\mu)-\omega(\xi)|^{p} d \mu \leq \mathcal{F}\left(\xi ; n, p, \iota_{1}, \iota_{2}\right) \max \left\{\left[\omega^{(n)}\left(\iota_{1}\right)\right]^{p},\left[\omega^{(n)}\left(\iota_{2}\right)\right]^{p}\right\},
$$

where $\mathcal{F}\left(\xi ; n, p, \iota_{1}, \iota_{2}\right)$ is defined as in Theorem 24.

Proof. From the quasi-convexity of $\left[\omega^{(n)}(\mu)\right]^{p}$ on $\left(\iota_{1}, \iota_{2}\right)$, we have:

$$
\begin{gathered}
\frac{1}{\iota_{2}-\iota_{1}} \int_{\iota_{1}}^{\iota_{2}}\left[\omega^{(n)}(\mu)\right]^{p} d \mu=\int_{0}^{1}\left[\omega^{(n)}\left(\delta \iota_{1}+(1-\delta) \iota_{2}\right)\right]^{p} d \delta \leq \int_{0}^{1} \max \left\{\left[\omega^{(n)}\left(\iota_{1}\right)\right]^{p},\left[\omega^{(n)}\left(\iota_{2}\right)\right]^{p}\right\} d \delta \\
=\max \left\{\left[\omega^{(n)}\left(\iota_{1}\right)\right]^{p},\left[\omega^{(n)}\left(\iota_{2}\right)\right]^{p}\right\} .
\end{gathered}
$$

Multiplying by $\mathcal{F}\left(\xi ; n, p, \iota_{1}, \iota_{2}\right)$ and using Theorem 4 , we obtain the desired inequality (28).

Theorem 26. Let $\xi \in\left(\iota_{1}, \iota_{2}\right)$ and $\omega$ be an $n$-times differentiable function on $\left(\iota_{1}, \iota_{2}\right)$, where $\omega, \omega^{\prime}, \ldots, \omega^{(n)}$ are positive with $\omega^{(j)}\left(\iota_{1}\right)=\omega^{(j)}\left(\iota_{2}\right)=0$, for all $j=0,1, \ldots, n-1$. If $\left[\omega^{(n)}(\mu)\right]^{p}$ is a convex function on $\left(\iota_{1}, \iota_{2}\right)$, then for all $p>1$, we have:

$$
\int_{\iota_{1}}^{\iota_{2}}|\omega(\mu)-\mathcal{\omega}(\xi)|^{p} d \mu \leq \mathcal{F}\left(\xi ; n, p, \iota_{1}, \iota_{2}\right)\left\{\frac{\left[\omega^{(n)}\left(\iota_{1}\right)\right]^{p}+\left[\omega^{(n)}\left(\iota_{2}\right)\right]^{p}}{2}\right\},
$$

where $\mathcal{F}\left(\xi ; n, p, \iota_{1}, \iota_{2}\right)$ is defined as in Theorem 24.

Proof. From the convexity of $\left[\omega^{(n)}(\mu)\right]^{p}$ on $\left(\iota_{1}, \iota_{2}\right)$, we have:

$$
\begin{gathered}
\frac{1}{\iota_{2}-\iota_{1}} \int_{\iota_{1}}^{\iota_{2}}\left[\omega^{(n)}(\mu)\right]^{p} d \mu=\int_{0}^{1}\left[\omega^{(n)}\left(\delta \iota_{1}+(1-\delta) \iota_{2}\right)\right]^{p} d \delta \\
\leq \int_{0}^{1}\left(\delta\left[\omega^{(n)}\left(\iota_{1}\right)\right]^{p}+(1-\delta)\left[\omega^{(n)}\left(\iota_{2}\right)\right]^{p}\right) d \delta \\
=\frac{\left[\omega^{(n)}\left(\iota_{1}\right)\right]^{p}+\left[\omega^{(n)}\left(\iota_{2}\right)\right]^{p}}{2} .
\end{gathered}
$$

Multiplying by $\mathcal{F}\left(\xi ; n, p, \iota_{1}, \iota_{2}\right)$ and using Theorem 4 , we obtain the desired inequality (29).

Theorem 27. Let $\xi \in\left(\iota_{1}, \iota_{2}\right)$ and $\omega$ be an $n$-times differentiable function on $\left(\iota_{1}, \iota_{2}\right)$, where $\omega, \omega^{\prime}, \ldots, \omega^{(n)}$ are positive with $\omega^{(j)}\left(\iota_{1}\right)=\omega^{(j)}\left(\iota_{2}\right)=0$, for all $j=0,1, \ldots, n-1$. If $\left[\omega^{(n)}(\mu)\right]^{p}$ is an s-convex function on $\left(\iota_{1}, \iota_{2}\right)$, then for all $p>1$, we have:

$$
\int_{\iota_{1}}^{\iota_{2}}|\omega(\mu)-\omega(\xi)|^{p} d \mu \leq \mathcal{F}\left(\xi ; n, p, \iota_{1}, \iota_{2}\right)\left\{\frac{\left[\omega^{(n)}\left(\iota_{1}\right)\right]^{p}+\left[\omega^{(n)}\left(\iota_{2}\right)\right]^{p}}{s+1}\right\},
$$

where $\mathcal{F}\left(\xi ; n, p, \iota_{1}, \iota_{2}\right)$ is defined as in Theorem 24. 
Proof. From the $s$-convexity of $\left[\omega^{(n)}(\mu)\right]^{p}$ on $\left(\iota_{1}, \iota_{2}\right)$, we have:

$$
\begin{gathered}
\frac{1}{\iota_{2}-\iota_{1}} \int_{\iota_{1}}^{\iota_{2}}\left[\omega^{(n)}(\mu)\right]^{p} d \mu=\int_{0}^{1}\left[\omega^{(n)}\left(\delta \iota_{1}+(1-\delta) \iota_{2}\right)\right]^{p} d \delta \\
\leq \int_{0}^{1}\left(\delta^{s}\left[\omega^{(n)}\left(\iota_{1}\right)\right]^{p}+(1-\delta)^{S}\left[\omega^{(n)}\left(\iota_{2}\right)\right]^{p}\right) d \delta \\
=\frac{\left[\omega^{(n)}\left(\iota_{1}\right)\right]^{p}+\left[\omega^{(n)}\left(\iota_{2}\right)\right]^{p}}{s+1} .
\end{gathered}
$$

Multiplying by $\mathcal{F}\left(\xi ; n, p, \iota_{1}, \iota_{2}\right)$ and using Theorem 4 , we obtain the desired inequality (30).

Remark 12. Taking $s=1$ in Theorem 27, we obtain Theorem 26.

Theorem 28. Let $\xi \in\left(\iota_{1}, \iota_{2}\right)$ and $\omega$ be an $n$-times differentiable function on $\left(\iota_{1}, \iota_{2}\right)$, where $\omega, \omega^{\prime}, \ldots, \omega^{(n)}$ are positive with $\omega^{(j)}\left(\iota_{1}\right)=\omega^{(j)}\left(\iota_{2}\right)=0$, for all $j=0,1, \ldots, n-1$. If $\left[\omega^{(n)}(\mu)\right]^{p}$ is an m-convex function on $\left(\iota_{1}, \iota_{2}\right)$, then for all $p>1$ and $m \in(0,1]$, we have:

$$
\begin{gathered}
\int_{\iota_{1}}^{\iota_{2}}|\omega(\mu)-\omega(\xi)|^{p} d \mu \leq \mathcal{F}\left(\xi ; n, p, \iota_{1}, \iota_{2}\right) \frac{\left(\iota_{1}-m \iota_{2}\right)}{\iota_{2}-\iota_{1}} \\
\times\left\{\frac{1}{2}\left[\left(\frac{\iota_{2}(1-m)}{\iota_{1}-m \iota_{2}}\right)^{2}-1\right]\left[\omega^{(n)}\left(\iota_{1}\right)\right]^{p}\right. \\
\left.+\left[m\left(\frac{\iota_{2}(1-m)}{\iota_{1}-m \iota_{2}}-1\right)-\frac{m}{2}\left(\left(\frac{\iota_{2}(1-m)}{\iota_{1}-m \iota_{2}}\right)^{2}-1\right)\right]\left[\omega^{(n)}\left(\iota_{2}\right)\right]^{p}\right\},
\end{gathered}
$$

where $\mathcal{F}\left(\xi ; n, p, \iota_{1}, \iota_{2}\right)$ is defined as in Theorem 24.

Proof. From the $m$-convexity of $\left[\omega^{(n)}(\mu)\right]^{p}$ on $\left(\iota_{1}, \iota_{2}\right)$, we have:

$$
\begin{gathered}
\frac{1}{\iota_{2}-\iota_{1}} \int_{\iota_{1}}^{\iota_{2}}\left[\omega^{(n)}(\mu)\right]^{p} d \mu=\frac{\left(\iota_{1}-m \iota_{2}\right)}{\iota_{2}-\iota_{1}} \int_{1}^{\frac{\iota_{2}(1-m)}{l_{1}-m \iota_{2}}}\left[\omega^{(n)}\left(\delta \iota_{1}+m(1-\delta) \iota_{2}\right)\right]^{p} d \delta \\
\leq \frac{\left(\iota_{1}-m \iota_{2}\right)}{\iota_{2}-\iota_{1}} \int_{1}^{\frac{\iota^{2}(1-m)}{\iota_{1}-m \iota_{2}}}\left(\delta\left[\omega^{(n)}\left(\iota_{1}\right)\right]^{p}+m(1-\delta)\left[\omega^{(n)}\left(\iota_{2}\right)\right]^{p}\right) d \delta \\
=\frac{\left(\iota_{1}-m \iota_{2}\right)}{\iota_{2}-\iota_{1}}\left\{\frac{1}{2}\left[\left(\frac{\iota_{2}(1-m)}{\iota_{1}-m \iota_{2}}\right)^{2}-1\right]\left[\omega^{(n)}\left(\iota_{1}\right)\right]^{p}\right. \\
\left.+\left[m\left(\frac{\iota_{2}(1-m)}{\iota_{1}-m \iota_{2}}-1\right)-\frac{m}{2}\left(\left(\frac{\iota_{2}(1-m)}{\iota_{1}-m \iota_{2}}\right)^{2}-1\right)\right]\left[\omega^{(n)}\left(\iota_{2}\right)\right]^{p}\right\} .
\end{gathered}
$$

Multiplying by $\mathcal{F}\left(\xi ; n, p, \iota_{1}, \iota_{2}\right)$ and using Theorem 4 , we obtain the desired inequality (31).

Remark 13. Taking $m=1$ in Theorem 28, we obtain Theorem 26.

Theorem 29. Let $\xi \in\left(\iota_{1}, \iota_{2}\right)$ and $\omega$ be an $n$-times differentiable function on $\left(\iota_{1}, \iota_{2}\right)$, where $\omega, \omega^{\prime}, \ldots, \omega^{(n)}$ are positive with $\omega^{(j)}\left(\iota_{1}\right)=\omega^{(j)}\left(\iota_{2}\right)=0$, for all $j=0,1, \ldots, n-1$. If $\left[\omega^{(n)}(\mu)\right]^{p}$ is an $(\alpha, m)$-convex function on $\left(\iota_{1}, \iota_{2}\right)$, then for all $p>1$ and $(\alpha, m) \in(0,1]^{2}$, we have:

$$
\begin{gathered}
\int_{\iota_{1}}^{\iota_{2}}|\omega(\mu)-\omega(\xi)|^{p} d \mu \leq \mathcal{F}\left(\xi ; n, p, \iota_{1}, \iota_{2}\right) \frac{\left(\iota_{1}-m \iota_{2}\right)}{\iota_{2}-\iota_{1}} \\
\times\left\{\frac{1}{\alpha+1}\left[\left(\frac{\iota_{2}(1-m)}{\iota_{1}-m \iota_{2}}\right)^{\alpha+1}-1\right]\left[\omega^{(n)}\left(\iota_{1}\right)\right]^{p}\right. \\
\left.+\left[m\left(\frac{\iota_{2}(1-m)}{\iota_{1}-m \iota_{2}}-1\right)-\frac{m}{\alpha+1}\left(\left(\frac{\iota_{2}(1-m)}{\iota_{1}-m \iota_{2}}\right)^{\alpha+1}-1\right)\right]\left[\omega^{(n)}\left(\iota_{2}\right)\right]^{p}\right\},
\end{gathered}
$$

where $\mathcal{F}\left(\xi ; n, p, \iota_{1}, \iota_{2}\right)$ is defined as in Theorem 24. 
Proof. From the $(\alpha, m)$-convexity of $\left[\omega^{(n)}(\mu)\right]^{p}$ on $\left(\iota_{1}, \iota_{2}\right)$, we have:

$$
\begin{gathered}
\frac{1}{\iota_{2}-\iota_{1}} \int_{\iota_{1}}^{\iota_{2}}\left[\mathcal{\omega}^{(n)}(\mu)\right]^{p} d \mu=\frac{\left(\iota_{1}-m \iota_{2}\right)}{\iota_{2}-\iota_{1}} \int_{1}^{\frac{\iota_{2}(1-m)}{\iota_{1}-m \iota_{2}}}\left[\omega^{(n)}\left(\delta \iota_{1}+m(1-\delta) \iota_{2}\right)\right]^{p} d \delta \\
\leq \frac{\left(\iota_{1}-m \iota_{2}\right)}{\iota_{2}-\iota_{1}} \int_{1}^{\frac{\iota_{2}(-m)}{l_{1}-m \iota_{2}}}\left(\delta^{\alpha}\left[\omega^{(n)}\left(\iota_{1}\right)\right]^{p}+m\left(1-\delta^{\alpha}\right)\left[\omega^{(n)}\left(\iota_{2}\right)\right]^{p}\right) d \delta \\
=\frac{\left(\iota_{1}-m \iota_{2}\right)}{\iota_{2}-\iota_{1}}\left\{\frac{1}{\alpha+1}\left[\left(\frac{\iota_{2}(1-m)}{\iota_{1}-m \iota_{2}}\right)^{\alpha+1}-1\right]\left[\omega^{(n)}\left(\iota_{1}\right)\right]^{p}\right. \\
\left.+\left[m\left(\frac{\iota_{2}(1-m)}{\iota_{1}-m \iota_{2}}-1\right)-\frac{m}{\alpha+1}\left(\left(\frac{\iota_{2}(1-m)}{\iota_{1}-m \iota_{2}}\right)^{\alpha+1}-1\right)\right]\left[\omega^{(n)}\left(\iota_{2}\right)\right]^{p}\right\} .
\end{gathered}
$$

Multiplying by $\mathcal{F}\left(\xi ; n, p, \iota_{1}, \iota_{2}\right)$ and using Theorem 4 , we obtain the desired inequality (32).

Remark 14. Taking $\alpha=1$ in Theorem 29, we obtain Theorem 28.

Theorem 30. Let $\iota_{1}, \iota_{2} \in I^{\circ}$, and $\iota_{1}<\iota_{2}$. Assume that $\omega$ is an $n$-times differentiable function on $\left(\iota_{1}, \iota_{2}\right)$, where $\omega, \omega^{\prime}, \ldots, \omega^{(n)}$ are positive with $\omega^{(j)}\left(\iota_{1}\right)=\omega^{(j)}\left(\iota_{2}\right)=0$, for all $j=0,1, \ldots, n-1$. If $\left[\omega^{(n)}(\mu)\right]^{p}$ is a P-convex function on $\left(\iota_{1}, \iota_{2}\right)$, then for all $p>1$, we have:

$$
\left|\omega(\mu)-\frac{1}{\iota_{2}-\iota_{1}} \int_{\iota_{1}}^{\iota_{2}} \omega(\delta) d \delta\right| \leq \sqrt[p]{\frac{\mathcal{F}\left(\xi ; n, p, \iota_{1}, \iota_{2}\right)\left\{\left[\omega^{(n)}\left(\iota_{1}\right)\right]^{p}+\left[\omega^{(n)}\left(\iota_{2}\right)\right]^{p}\right\}}{\iota_{2}-\iota_{1}}},
$$

where $\mathcal{F}\left(\xi ; n, p, \iota_{1}, \iota_{2}\right)$ is defined as in Theorem 24

Proof. It is obvious that:

$$
\omega(\mu)-\frac{1}{\iota_{2}-\iota_{1}} \int_{\iota_{1}}^{\iota_{2}} \omega(\delta) d \delta=\frac{1}{\iota_{2}-\iota_{1}} \int_{\iota_{1}}^{\iota_{2}}[\omega(\mu)-\omega(\delta)] d \delta .
$$

Taking the modulus, applying the triangle inequality, and then, using the Hölder inequality, we obtain:

$$
\begin{aligned}
& \left|\omega(\mu)-\frac{1}{\iota_{2}-\iota_{1}} \int_{\iota_{1}}^{\iota_{2}} \omega(\delta) d \delta\right|=\left|\frac{1}{\iota_{2}-\iota_{1}} \int_{\iota_{1}}^{\iota_{2}}[\omega(\mu)-\omega(\delta)] d \delta\right| \\
& \leq \frac{1}{\iota_{2}-\iota_{1}} \int_{\iota_{1}}^{\iota_{2}}|\omega(\mu)-\omega(\delta)| d \delta \leq \frac{1}{\sqrt[p]{\iota_{2}-\iota_{1}}}\left(\int_{\iota_{1}}^{\iota_{2}}|\omega(\mu)-\omega(\delta)|^{p} d \delta\right)^{\frac{1}{p}}
\end{aligned}
$$

From the $P$-convexity of $\left[\mathcal{O}^{(n)}(\mu)\right]^{p}$ on $\left(\iota_{1}, \iota_{2}\right)$ and applying Theorem 24 , we obtain the desired inequality (33).

Theorem 31. Let $\iota_{1}, \iota_{2} \in I^{\circ}$, and $\iota_{1}<\iota_{2}$. Suppose that $\omega$ is an $n$-times differentiable function on $\left(\iota_{1}, \iota_{2}\right)$, where $\omega, \omega^{\prime}, \ldots, \omega^{(n)}$ are positive with $\omega^{(j)}\left(\iota_{1}\right)=\omega^{(j)}\left(\iota_{2}\right)=0$, for all $j=0,1, \ldots, n-1$. If $\left[\omega^{(n)}(\mu)\right]^{p}$ is a quasi-convex function on $\left(\iota_{1}, \iota_{2}\right)$, then for all $p>1$, we have:

$$
\left|\omega(\mu)-\frac{1}{\iota_{2}-\iota_{1}} \int_{\iota_{1}}^{\iota_{2}} \omega(\delta) d \delta\right| \leq \sqrt[p]{\frac{\mathcal{F}\left(\xi ; n, p, \iota_{1}, \iota_{2}\right) \max \left\{\left[\omega^{(n)}\left(\iota_{1}\right)\right]^{p},\left[\omega^{(n)}\left(\iota_{2}\right)\right]^{p}\right\}}{\iota_{2}-\iota_{1}}},
$$

where $\mathcal{F}\left(\xi ; n, p, \iota_{1}, \iota_{2}\right)$ is defined as in Theorem 24. 
Proof. From Inequality (34), the quasi-convexity of $\left[\omega^{(n)}(\mu)\right]^{p}$ on $\left(\iota_{1}, \iota_{2}\right)$, and applying Theorem 25, we obtain the desired inequality (35).

Theorem 32. Let $\iota_{1}, \iota_{2} \in I^{\circ}$, and $\iota_{1}<\iota_{2}$. Assume that $\omega$ is an $n$-times differentiable function on $\left(\iota_{1}, \iota_{2}\right)$, where $\omega, \omega^{\prime}, \ldots, \omega^{(n)}$ are positive with $\omega^{(j)}\left(\iota_{1}\right)=\omega^{(j)}\left(\iota_{2}\right)=0$, for all $j=0,1, \ldots, n-1$. If $\left[\omega^{(n)}(\mu)\right]^{p}$ is a convex function on $\left(\iota_{1}, \iota_{2}\right)$, then for all $p>1$, we have:

$$
\left|\omega(\mu)-\frac{1}{\iota_{2}-\iota_{1}} \int_{\iota_{1}}^{\iota_{2}} \omega(\delta) d \delta\right| \leq \sqrt[p]{\frac{\mathcal{F}\left(\xi ; n, p, \iota_{1}, \iota_{2}\right)\left\{\left[\omega^{(n)}\left(\iota_{1}\right)\right]^{p}+\left[\omega^{(n)}\left(\iota_{2}\right)\right]^{p}\right\}}{2\left(\iota_{2}-\iota_{1}\right)}},
$$

where $\mathcal{F}\left(\xi ; n, p, \iota_{1}, \iota_{2}\right)$ is defined as in Theorem 24.

Proof. From Inequality (34), the convexity of $\left[\omega^{(n)}(\mu)\right]^{p}$ on $\left(\iota_{1}, \iota_{2}\right)$, and applying Theorem 26, we obtain the desired inequality (36).

Theorem 33. Let $\iota_{1}, \iota_{2} \in I^{\circ}$, and $\iota_{1}<\iota_{2}$. Suppose that $\omega$ is an $n$-times differentiable function on $\left(\iota_{1}, \iota_{2}\right)$, where $\omega^{p}, \omega^{\prime}, \ldots, \omega^{(n)}$ are positive with $\omega^{(j)}\left(\iota_{1}\right)=\omega^{(j)}\left(\iota_{2}\right)=0$, for all $j=0,1, \ldots, n-1$. If $\left[\mathfrak{\omega}^{(n)}(\mu)\right]^{p}$ is an s-convex function on $\left(\iota_{1}, \iota_{2}\right)$, then for all $p>1$, we have:

$$
\left|\omega(\mu)-\frac{1}{\iota_{2}-\iota_{1}} \int_{\iota_{1}}^{\iota_{2}} \mathscr{\omega}(\delta) d \delta\right| \leq \sqrt[p]{\frac{\mathcal{F}\left(\xi ; n, p, \iota_{1}, \iota_{2}\right)\left\{\left[\omega^{(n)}\left(\iota_{1}\right)\right]^{p}+\left[\omega^{(n)}\left(\iota_{2}\right)\right]^{p}\right\}}{(s+1)\left(\iota_{2}-\iota_{1}\right)}},
$$

where $\mathcal{F}\left(\xi ; n, p, \iota_{1}, \iota_{2}\right)$ is defined as in Theorem 24.

Proof. From Inequality (34), the s-convexity of $\left[\omega^{(n)}(\mu)\right]^{p}$ on $\left(\iota_{1}, \iota_{2}\right)$, and applying Theorem 27, we obtain the desired inequality (37).

Remark 15. Taking $s=1$ in Theorem 33, we obtain Theorem 32.

Theorem 34. Let $\iota_{1}, l_{2} \in I^{\circ}$, and $\iota_{1}<\iota_{2}$. Assume that $\omega$ is an $n$-times differentiable function on $\left(\iota_{1}, \iota_{2}\right)$, where $\omega, \omega^{\prime}, \ldots, \omega^{(n)}$ are positive with $\omega^{(j)}\left(\iota_{1}\right)=\omega^{(j)}\left(\iota_{2}\right)=0$, for all $j=0,1, \ldots, n-1$. If $\left[\omega^{(n)}(\mu)\right]^{p}$ is an m-convex function on $\left(\iota_{1}, \iota_{2}\right)$, then for all $p>1$ and $m \in(0,1]$, we have:

$$
\begin{aligned}
& \left|\omega(\mu)-\frac{1}{\iota_{2}-l_{1}} \int_{l_{1}}^{\iota_{2}} \omega(\delta) d \delta\right| \leq \sqrt[p]{\frac{\mathcal{F}\left(\xi ; n, p, l_{1}, l_{2}\right)}{\iota_{2}-l_{1}}} \\
& \times\left[\frac { ( \iota _ { 1 } - m \iota _ { 2 } ) } { \iota _ { 2 } - \iota _ { 1 } } \left\{\frac{1}{2}\left[\left(\frac{\iota_{2}(1-m)}{\iota_{1}-m \iota_{2}}\right)^{2}-1\right]\left[\mathfrak{W}^{(n)}\left(\iota_{1}\right)\right]^{p}\right.\right. \\
& \left.\left.+\left[m\left(\frac{\iota_{2}(1-m)}{\iota_{1}-m l_{2}}-1\right)-\frac{m}{2}\left(\left(\frac{\iota_{2}(1-m)}{\iota_{1}-m \iota_{2}}\right)^{2}-1\right)\right]\left[\omega^{(n)}\left(\iota_{2}\right)\right]^{p}\right\}\right]^{\frac{1}{p}},
\end{aligned}
$$

where $\mathcal{F}\left(\xi ; n, p, \iota_{1}, \iota_{2}\right)$ is defined as in Theorem 24.

Proof. From Inequality (34), the $m$-convexity of $\left[\mathfrak{\omega}^{(n)}(\mu)\right]^{p}$ on $\left(\iota_{1}, \iota_{2}\right)$, and applying Theorem 28, we obtain the desired inequality (38).

Remark 16. Taking $m=1$ in Theorem 34, we obtain Theorem 32.

Theorem 35. Let $\iota_{1}, \iota_{2} \in I^{\circ}$, and $\iota_{1}<\iota_{2}$. Suppose that $\omega$ is an $n$-times differentiable function on $\left(\iota_{1}, \iota_{2}\right)$, where $\omega, \omega^{\prime}, \ldots, \omega^{(n)}$ are positive with $\omega^{(j)}\left(\iota_{1}\right)=\omega^{(j)}\left(\iota_{2}\right)=0$, for all $j=0,1, \ldots, n-1$. 
If $\left[\omega^{(n)}(\mu)\right]^{p}$ is an $(\alpha, m)$-convex function on $\left(\iota_{1}, \iota_{2}\right)$, then for all $p>1$ and $(\alpha, m) \in(0,1]^{2}$, we have:

$$
\begin{gathered}
\left|\mathcal{\omega}(\mu)-\frac{1}{\iota_{2}-\iota_{1}} \int_{\iota_{1}}^{\iota_{2}} \omega(\delta) d \delta\right| \leq \sqrt[p]{\frac{\mathcal{F}\left(\xi ; n, p, \iota_{1}, \iota_{2}\right)}{\iota_{2}-\iota_{1}}} \\
\times\left[\frac { ( \iota _ { 1 } - m \iota _ { 2 } ) } { \iota _ { 2 } - \iota _ { 1 } } \left\{\frac{1}{\alpha+1}\left[\left(\frac{\iota_{2}(1-m)}{\iota_{1}-m \iota_{2}}\right)^{\alpha+1}-1\right]\left[\omega^{(n)}\left(\iota_{1}\right)\right]^{p}\right.\right. \\
\left.\left.+\left[m\left(\frac{\iota_{2}(1-m)}{\iota_{1}-m \iota_{2}}-1\right)-\frac{m}{\alpha+1}\left(\left(\frac{\iota_{2}(1-m)}{\iota_{1}-m \iota_{2}}\right)^{\alpha+1}-1\right)\right]\left[\omega^{(n)}\left(\iota_{2}\right)\right]^{p}\right\}\right]^{\frac{1}{p}},
\end{gathered}
$$

where $\mathcal{F}\left(\xi ; n, p, \iota_{1}, \iota_{2}\right)$ is defined as in Theorem 24.

Proof. From Inequality (34), the $(\alpha, m)$-convexity of $\left[\omega^{(n)}(\mu)\right]^{p}$ on $\left(\iota_{1}, \iota_{2}\right)$, and applying Theorem 29, we obtain the desired inequality (39).

Remark 17. Taking $\alpha=1$ in Theorem 35, we obtain Theorem 34.

\section{Inequalities of the Chebyshev Type}

Theorem 36. Let $\iota_{1}, \iota_{2} \in I^{\circ}$, and $\iota_{1}<\iota_{2}$. Assume that $\omega, \vartheta$ are $n$-times differentiable functions on $\left(\iota_{1}, \iota_{2}\right)$, where $\omega, \omega^{\prime}, \ldots, \omega^{(n)}$ and $\vartheta, \vartheta^{\prime}, \ldots, \vartheta^{(n)}$ are positive with $\omega^{(j)}\left(\iota_{1}\right)=\omega^{(j)}\left(\iota_{2}\right)=0$, and $\vartheta^{(j)}\left(\iota_{1}\right)=\vartheta^{(j)}\left(\iota_{2}\right)=0$, for all $j=0,1, \ldots, n-1$. If $\left[\omega^{(n)}(\mu)\right]^{p}$ and $\left[\vartheta^{(n)}(\mu)\right]^{q}$ are P-convex functions on $\left(\iota_{1}, \iota_{2}\right)$, then for all $p>1$ such that $\frac{1}{p}+\frac{1}{q}=1$, we have:

$$
\begin{aligned}
|\mathcal{T}(\omega, \vartheta)| & \leq \frac{1}{\iota_{2}-\iota_{1}} \sqrt[p]{\mathcal{F}\left(\xi ; n, p, \iota_{1}, \iota_{2}\right)\left\{\left[\omega^{(n)}\left(\iota_{1}\right)\right]^{p}+\left[\omega^{(n)}\left(\iota_{2}\right)\right]^{p}\right\}}, \\
& \times \sqrt[q]{\mathcal{F}\left(\xi ; n, q, \iota_{1}, \iota_{2}\right)\left\{\left[\vartheta^{(n)}\left(\iota_{1}\right)\right]^{q}+\left[\vartheta^{(n)}\left(\iota_{2}\right)\right]^{q}\right\}},
\end{aligned}
$$

where $\mathcal{F}\left(\xi ; n, p, \iota_{1}, \iota_{2}\right)$ is defined as in Theorem 24.

Proof. From the equality:

$$
\mathcal{T}(\omega, \vartheta)=\frac{1}{\iota_{2}-\iota_{1}} \int_{\iota_{1}}^{\iota_{2}}\left[\omega(\mu)-\frac{1}{\iota_{2}-\iota_{1}} \int_{\iota_{1}}^{\iota_{2}} \omega(\delta) d \delta\right]\left[\vartheta(\mu)-\frac{1}{\iota_{2}-\iota_{1}} \int_{\iota_{1}}^{\iota_{2}} \vartheta(\delta) d \delta\right] d \mu,
$$

taking the absolute value, and then, applying the Cauchy-Schwartz inequality, we obtain:

$$
\begin{gathered}
|\mathcal{T}(\omega, \vartheta)| \leq \frac{1}{\iota_{2}-\iota_{1}}\left(\int_{\iota_{1}}^{\iota_{2}}\left|\omega(\mu)-\frac{1}{\iota_{2}-\iota_{1}} \int_{\iota_{1}}^{\iota_{2}} \omega(\delta) d \delta\right|^{2} d \mu\right)^{\frac{1}{2}} \\
\times\left(\int_{\iota_{1}}^{\iota_{2}}\left|\vartheta(\mu)-\frac{1}{\iota_{2}-\iota_{1}} \int_{\iota_{1}}^{\iota_{2}} \vartheta(\delta) d \delta\right|^{2} d \mu\right)^{\frac{1}{2}} .
\end{gathered}
$$

From the $P$-convexity of functions $\left[\omega^{(n)}(\mu)\right]^{p}$ and $\left[\vartheta^{(n)}(\mu)\right]^{q}$ on $\left(\iota_{1}, \iota_{2}\right)$ and applying Theorem 30, we obtain the desired inequality (40).

Theorem 37. Let $\iota_{1}, \iota_{2} \in I^{\circ}$, and $\iota_{1}<\iota_{2}$. Suppose that $\omega, \vartheta$ are $n$-times differentiable functions on $\left(\iota_{1}, \iota_{2}\right)$, where $\omega, \omega^{\prime}, \ldots, \omega^{(n)}$ and $\vartheta, \vartheta^{\prime}, \ldots, \vartheta^{(n)}$ are positive with $\omega^{(j)}\left(\iota_{1}\right)=\omega^{(j)}\left(\iota_{2}\right)=0$, and $\vartheta^{(j)}\left(\iota_{1}\right)=\vartheta^{(j)}\left(\iota_{2}\right)=0$, for all $j=0,1, \ldots, n-1$. If $\left[\omega^{(n)}(\mu)\right]^{p}$ and $\left[\vartheta^{(n)}(\mu)\right]^{q}$ are quasi-convex functions on $\left(\iota_{1}, \iota_{2}\right)$, then for all $p>1$ such that $\frac{1}{p}+\frac{1}{q}=1$, we have: 


$$
\begin{aligned}
|\mathcal{T}(\omega, \vartheta)| & \leq \frac{1}{\iota_{2}-\iota_{1}} \sqrt[p]{\mathcal{F}\left(\xi ; n, p, \iota_{1}, \iota_{2}\right) \max \left\{\left[\omega^{(n)}\left(\iota_{1}\right)\right]^{p},\left[\omega^{(n)}\left(\iota_{2}\right)\right]^{p}\right\}}, \\
& \times \sqrt[q]{\mathcal{F}\left(\xi ; n, q, \iota_{1}, \iota_{2}\right) \max \left\{\left[\vartheta^{(n)}\left(\iota_{1}\right)\right]^{q},\left[\vartheta^{(n)}\left(\iota_{2}\right)\right]^{q}\right\}},
\end{aligned}
$$

where $\mathcal{F}\left(\xi ; n, p, \iota_{1}, \iota_{2}\right)$ is defined as in Theorem 24.

Proof. From Inequality (41), the quasi-convexity of functions $\left[\mathcal{O}^{(n)}(\mu)\right]^{p}$ and $\left[\vartheta^{(n)}(\mu)\right]^{q}$ on $\left(\iota_{1}, \iota_{2}\right)$, and applying Theorem 31, we obtain the desired inequality (42).

Theorem 38. Let $\iota_{1}, \iota_{2} \in I^{\circ}$, and $\iota_{1}<\iota_{2}$. Assume that $\omega, \vartheta$ are $n$-times differentiable functions on $\left(\iota_{1}, \iota_{2}\right)$, where $\omega, \omega^{\prime}, \ldots, \omega^{(n)}$ and $\vartheta, \vartheta^{\prime}, \ldots, \vartheta^{(n)}$ are positive with $\omega^{(j)}\left(\iota_{1}\right)=\omega^{(j)}\left(\iota_{2}\right)=0$, and $\vartheta^{(j)}\left(\iota_{1}\right)=\vartheta^{(j)}\left(\iota_{2}\right)=0$, for all $j=0,1, \ldots, n-1$. If $\left[\omega^{(n)}(\mu)\right]^{p}$ and $\left[\vartheta^{(n)}(\mu)\right]^{q}$ are convex functions on $\left(\iota_{1}, \iota_{2}\right)$, then for all $p>1$ such that $\frac{1}{p}+\frac{1}{q}=1$, we have:

$$
\begin{aligned}
|\mathcal{T}(\omega, \vartheta)| & \leq \frac{1}{2\left(\iota_{2}-\iota_{1}\right)} \sqrt[p]{\mathcal{F}\left(\xi ; n, p, \iota_{1}, \iota_{2}\right)\left(\left[\omega^{(n)}\left(\iota_{1}\right)\right]^{p}+\left[\omega^{(n)}\left(\iota_{2}\right)\right]^{p}\right)}, \\
& \times \sqrt[q]{\mathcal{F}\left(\xi ; n, q, \iota_{1}, \iota_{2}\right)\left(\left[\vartheta^{(n)}\left(\iota_{1}\right)\right]^{q}+\left[\vartheta^{(n)}\left(\iota_{2}\right)\right]^{q}\right)},
\end{aligned}
$$

where $\mathcal{F}\left(\xi ; n, p, \iota_{1}, \iota_{2}\right)$ is defined as in Theorem 24.

Proof. From Inequality (41), the convexity of functions $\left[\omega^{(n)}(\mu)\right]^{p}$ and $\left[\vartheta^{(n)}(\mu)\right]^{q}$ on $\left(\iota_{1}, \iota_{2}\right)$, and applying Theorem 32, we obtain the desired inequality (43).

Theorem 39. Let $\iota_{1}, \iota_{2} \in I^{\circ}$, and $\iota_{1}<\iota_{2}$. Suppose that $\omega, \vartheta$ are $n$-times differentiable functions on $\left(\iota_{1}, \iota_{2}\right)$, where $\omega, \omega^{\prime}, \ldots, \omega^{(n)}$ and $\vartheta, \vartheta^{\prime}, \ldots, \vartheta^{(n)}$ are positive with $\omega^{(j)}\left(\iota_{1}\right)=\omega^{(j)}\left(\iota_{2}\right)=0$, and $\vartheta^{(j)}\left(\iota_{1}\right)=\vartheta^{(j)}\left(\iota_{2}\right)=0$, for all $j=0,1, \ldots, n-1$. If $\left[\omega^{(n)}(\mu)\right]^{p}$ and $\left[\vartheta^{(n)}(\mu)\right]^{q}$ are s-convex functions on $\left(\iota_{1}, \iota_{2}\right)$, then for all $p>1$ such that $\frac{1}{p}+\frac{1}{q}=1$, we have:

$$
\begin{aligned}
|\mathcal{T}(\omega, \vartheta)| \leq & \frac{1}{(s+1)\left(\iota_{2}-\iota_{1}\right)} \sqrt[p]{\mathcal{F}\left(\xi ; n, p, \iota_{1}, \iota_{2}\right)\left(\left[\omega^{(n)}\left(\iota_{1}\right)\right]^{p}+\left[\omega^{(n)}\left(\iota_{2}\right)\right]^{p}\right)}, \\
& \times \sqrt[q]{\mathcal{F}\left(\xi ; n, q, \iota_{1}, \iota_{2}\right)\left(\left[\vartheta^{(n)}\left(\iota_{1}\right)\right]^{q}+\left[\vartheta^{(n)}\left(\iota_{2}\right)\right]^{q}\right)},
\end{aligned}
$$

where $\mathcal{F}\left(\xi ; n, p, \iota_{1}, \iota_{2}\right)$ is defined as in Theorem 24.

Proof. From Inequality (41), the s-convexity of functions $\left[\mathcal{O}^{(n)}(\mu)\right]^{p}$ and $\left[\vartheta^{(n)}(\mu)\right]^{q}$ on $\left(\iota_{1}, \iota_{2}\right)$, and applying Theorem 33 , we obtain the desired inequality $(44)$.

Remark 18. Taking $s=1$ in Theorem 39, we obtain Theorem 38.

Theorem 40. Let $\iota_{1}, \iota_{2} \in I^{\circ}$, and $\iota_{1}<\iota_{2}$. Assume that $\omega, \vartheta$ are $n$-times differentiable functions on $\left(\iota_{1}, \iota_{2}\right)$, where $\omega, \omega^{\prime}, \ldots, \omega^{(n)}$ and $\vartheta, \vartheta^{\prime}, \ldots, \vartheta^{(n)}$ are positive with $\omega^{(j)}\left(\iota_{1}\right)=\omega^{(j)}\left(\iota_{2}\right)=0$, and $\vartheta^{(j)}\left(\iota_{1}\right)=\vartheta^{(j)}\left(\iota_{2}\right)=0$, for all $j=0,1, \ldots, n-1$. If $\left[\omega^{(n)}(\mu)\right]^{p}$ and $\left[\vartheta^{(n)}(\mu)\right]^{q}$ are $m$-convex functions on $\left(\iota_{1}, \iota_{2}\right)$, then for all $p>1$ and $m \in(0,1]$ such that $\frac{1}{p}+\frac{1}{q}=1$, we have: 


$$
\begin{gathered}
|\mathcal{T}(\omega, \vartheta)| \leq \frac{\sqrt[p]{\mathcal{F}\left(\xi ; n, p, \iota_{1}, \iota_{2}\right)} \sqrt[q]{\mathcal{F}\left(\xi ; n, q, \iota_{1}, \iota_{2}\right)}}{\iota_{2}-\iota_{1}} \\
\times\left[\frac { ( \iota _ { 1 } - m \iota _ { 2 } ) } { \iota _ { 2 } - \iota _ { 1 } } \left\{\frac{1}{2}\left[\left(\frac{\iota_{2}(1-m)}{\iota_{1}-m \iota_{2}}\right)^{2}-1\right]\left[\omega^{(n)}\left(\iota_{1}\right)\right]^{p}\right.\right. \\
\left.\left.+\left[m\left(\frac{\iota_{2}(1-m)}{\iota_{1}-m \iota_{2}}-1\right)-\frac{m}{2}\left(\left(\frac{\iota_{2}(1-m)}{\iota_{1}-m \iota_{2}}\right)^{2}-1\right)\right]\left[\omega^{(n)}\left(\iota_{2}\right)\right]^{p}\right\}\right]^{\frac{1}{p}} \\
\times\left[\frac { ( \iota _ { 1 } - m \iota _ { 2 } ) } { \iota _ { 2 } - \iota _ { 1 } } \left\{\frac{1}{2}\left[\left(\frac{\iota_{2}(1-m)}{\iota_{1}-m \iota_{2}}\right)^{2}-1\right]\left[\vartheta^{(n)}\left(\iota_{1}\right)\right]^{q}\right.\right. \\
\left.\left.+\left[m\left(\frac{\iota_{2}(1-m)}{\iota_{1}-m \iota_{2}}-1\right)-\frac{m}{2}\left(\left(\frac{\iota_{2}(1-m)}{\iota_{1}-m \iota_{2}}\right)^{2}-1\right)\right]\left[\vartheta^{(n)}\left(\iota_{2}\right)\right]^{q}\right\}\right]^{\frac{1}{q}},
\end{gathered}
$$

where $\mathcal{F}\left(\xi ; n, p, \iota_{1}, \iota_{2}\right)$ is defined as in Theorem 24.

Proof. From Inequality (41), the $m$-convexity of functions $\left[\omega^{(n)}(\mu)\right]^{p}$ and $\left[\vartheta^{(n)}(\mu)\right]^{q}$ on $\left(\iota_{1}, \iota_{2}\right)$, and applying Theorem 34 , we obtain the desired inequality (45).

Remark 19. Taking $m=1$ in Theorem 40, we obtain Theorem 38 .

Theorem 41. Let $\iota_{1}, \iota_{2} \in I^{\circ}$, and $\iota_{1}<\iota_{2}$. Suppose that $\omega, \vartheta$ are $n$-times differentiable functions on $\left(\iota_{1}, \iota_{2}\right)$, where $\omega, \omega^{\prime}, \ldots, \omega^{(n)}$ and $\vartheta, \vartheta^{\prime}, \ldots, \vartheta^{(n)}$ are positive with $\omega^{(j)}\left(\iota_{1}\right)=\omega^{(j)}\left(\iota_{2}\right)=0$, and $\vartheta^{(j)}\left(\iota_{1}\right)=\vartheta^{(j)}\left(\iota_{2}\right)=0$, for all $j=0,1, \ldots, n-1$. If $\left[\omega^{(n)}(\mu)\right]^{p}$ and $\left[\vartheta^{(n)}(\mu)\right]^{q}$ are $(\alpha, m)$ convex functions on $\left(\iota_{1}, \iota_{2}\right)$, then for all $p>1$ and $(\alpha, m) \in(0,1]^{2}$ such that $\frac{1}{p}+\frac{1}{q}=1$, we have:

$$
\begin{gathered}
|\mathcal{T}(\omega, \vartheta)| \leq \frac{\sqrt[p]{\mathcal{F}\left(\xi ; n, p, \iota_{1}, \iota_{2}\right)} \sqrt[V]{\mathcal{F}\left(\xi ; n, q, \iota_{1}, \iota_{2}\right)}}{\iota_{2}-\iota_{1}} \\
\times\left[\frac { ( \iota _ { 1 } - m \iota _ { 2 } ) } { \iota _ { 2 } - \iota _ { 1 } } \left\{\frac{1}{\alpha+1}\left[\left(\frac{\iota_{2}(1-m)}{\iota_{1}-m \iota_{2}}\right)^{\alpha+1}-1\right]\left[\omega^{(n)}\left(\iota_{1}\right)\right]^{p}\right.\right. \\
\left.\left.+\left[m\left(\frac{\iota_{2}(1-m)}{\iota_{1}-m \iota_{2}}-1\right)-\frac{m}{\alpha+1}\left(\left(\frac{\iota_{2}(1-m)}{\iota_{1}-m \iota_{2}}\right)^{\alpha+1}-1\right)\right]\left[\omega^{(n)}\left(\iota_{2}\right)\right]^{p}\right\}\right]^{\frac{1}{p}} \\
\times\left[\frac { ( \iota _ { 1 } - m \iota _ { 2 } ) } { \iota _ { 2 } - \iota _ { 1 } } \left\{\frac{1}{\alpha+1}\left[\left(\frac{\iota_{2}(1-m)}{\iota_{1}-m \iota_{2}}\right)^{\alpha+1}-1\right]\left[\vartheta^{(n)}\left(\iota_{1}\right)\right]^{q}\right.\right. \\
\left.\left.+\left[m\left(\frac{\iota_{2}(1-m)}{\iota_{1}-m \iota_{2}}-1\right)-\frac{m}{\alpha+1}\left(\left(\frac{\iota_{2}(1-m)}{\iota_{1}-m \iota_{2}}\right)^{\alpha+1}-1\right)\right]\left[\vartheta^{(n)}\left(\iota_{2}\right)\right]^{q}\right\}\right]^{\frac{1}{q}},
\end{gathered}
$$

where $\mathcal{F}\left(\xi ; n, p, \iota_{1}, \iota_{2}\right)$ is defined as in Theorem 24.

Proof. From Inequality $(41)$, the $(\alpha, m)$-convexity of functions $\left[\omega^{(n)}(\mu)\right]^{p}$ and $\left[\vartheta^{(n)}(\mu)\right]^{q}$ on $\left(\iota_{1}, \iota_{2}\right)$, and applying Theorem 35 , we obtain the desired inequality (46).

Remark 20. Taking $\alpha=1$ in Theorem 41, we obtain Theorem 40.

\section{Conclusions}

In this paper, via different kinds of differentiable convex functions, some new inequalities of the Beesack-Wirtinger type were proven. Furthermore, we generalized our results for functions that are $n$-times differentiable convex. Finally, many interesting Ostrowskiand Chebyshev-type inequalities were derived as well. It is worth mentioning that from our results, several interesting inequalities using special means, modified Bessel functions of the first and second kind, $q$-digamma function where $q \in(0,1)$, and some error estimations for quadrature formulas can be found; see [15,32-37] for details. Since the different kinds 
of convex functions that we used to obtain our results have large applications in many mathematical areas, then they can be applied to derive several new important results in convex analysis, quantum mechanics, and related optimization theory and may stimulate further research in different areas of pure and applied sciences. Studies relating convexity may have useful applications in interdisciplinary studies, such as maximizing the likelihood from multiple linear regressions involving the Gauss-Laplace distribution. For more details, see [38-45].

Author Contributions: Conceptualization, A.K. and G.R.; methodology, A.K. and M.S.; software M.S. and Z.A.K.; validation, G.R. and Z.A.K.; formal analysis, A.K. and M.S.; investigation, A.K. and G.R.; resources, Z.A.K.; data curation, Z.A.K.; writing-original draft preparation, A.K. and M.S.; writing-review and editing, G.R. and Z.A.K.; visualization, Z.A.K.; supervision, Z.A.K.; project administration, Z.A.K.; funding acquisition, Z.A.K. All authors have read and agreed to the published version of the manuscript.

Funding: This research received no external funding.

Institutional Review Board Statement: Not applicable.

Informed Consent Statement: Not applicable.

Data Availability Statement: Not applicable.

Acknowledgments: Princess Nourah bint Abdulrahman University Researchers Supporting Project number (PNURSP2022R8), Princess Nourah bint Abdulrahman University, Riyadh, Saudi Arabia.

Conflicts of Interest: The authors declare that there are no conflicts of interest regarding the publication of this paper.

\section{References}

1. Blaschke, W. Kreis und Kuge; Verlag von Veit \& Comp.: Leipzig, Germany, 1916; 188p.

2. Coles, W.J. Wirtinger-type integral inequalities. Pac. J. Math. 1961, 11, 871-877. [CrossRef]

3. Erden, S. Wirtinger type inequalities for higher order differentiable functions. Turk. J. Math. 2020, 44, 656-661. [CrossRef]

4. Agarwal, R.P.; Bohner, M.; O'Regan, D.; Saker, S.H. Some dynamic Wirtinger-type inequalities and their applications. Pac. J. Math. 2011, 252, 1-18. [CrossRef]

5. Zhao, C.J. On Opial-Wirtinger type inequalities. AIMS Math. 2020, 5, 1275-1283. [CrossRef]

6. Beesack, P.R. Integral inequalities involving a function and its derivative. Am. Math. Mon. 1971, 78, 705-741. [CrossRef]

7. Beesack, P.R. Extensions of Wirtinger's inequality. Trans. R. Soc. Can. 1959, 53, 21-30.

8. Chebyshev, P.L. Sur les expressions approximatives des integrales definies par les autres prises entre les mêmes limites. Proc. Math. Soc. Charkov. 1882, 2, 93-98.

9. Alomari, M.W. On Beesack-Wirtinger inequality. Result. Math. 2017, 72, 1213-1225. [CrossRef]

10. Maširević, D.J.; Pogány, T.G. Bounds on Čebyšev functional for $C_{\varphi}[0,1]$ function class. J. Anal. 2014, 22, 1-30.

11. Rahman, G.; Nisar, K.S.; Abdeljawad, T. Certain new proportional and Hadamard proportional fractional integral inequalities. J. Inequal. Appl. 2021, 2021, 14. [CrossRef]

12. Khan, K.; Khan, Z.A.; Ali, A.; Irfan, M. Investigation of Hirota equation: Modified double Laplace decomposition method. Phys. Scr. 2021, 9, 1-15. [CrossRef]

13. Rahman, G.; Nisar, K.S.; Khan, S.U.; Baleanu, D.; Vijayakumar, V. On the weighted fractional integral inequalities for Chebyshev functionals. Adv. Differ. Equ. 2021, 2021, 19. [CrossRef]

14. Khan, Z.A.; Ahmad, I.; Shah, K. Applications of fixed point theory to investigate a system of fractional order differential equations. J. Funct. Spaces 2021, 2021, 1399764. [CrossRef]

15. Ayub, U.; Mubeen, S.; Abdeljawad, T.; Rahman, G.; Nisar, K.S. The new Mittag-Leffler function and its applications. J. Math. 2021, 2020, 2463782. [CrossRef]

16. Iqbal, S.; Samraiz, S.; Abdeljawad, T.; Nisar, K.S.; Rahman, G.; Khan, M.A. New generalized Pólya-Szegö and Čebyšev type inequalities with general kernel and measure. Adv. Differ. Equ. 2020, 2020, 20. [CrossRef]

17. Gul, R.; Shah, K.; Khan, Z.A.; Jarad, F. On a class of boundary value problems under ABC fractional derivative. Adv. Differ. Equ. 2021, 2021, 437. [CrossRef]

18. Nisar, K.S.; Rahman, G.; Baleanu, B.; Samraiz, M.; Iqbal, S. On the weighted fractional Pólya-Szegö and Čebyšev-types integral inequalities concerning another function. Adv. Differ. Equ. 2020, 2020, 18. [CrossRef]

19. Khan, Z.A.; Gul, R.; Shah, K. On impulsive boundary value problem with Riemann-Liouville fractional order derivative. J. Funct. Spaces 2021, 2021, 8331731. [CrossRef] 
20. Rahman, G.; Nisar, K.S.; Ghanbari, B.; Abdeljawad, T. On generalized fractional integral inequalities for the monotone weighted Chebyshev functionals. Adv. Differ. Equ. 2020, 2020, 19. [CrossRef]

21. Srivastava, H.M.; Kashuri, A.; Mohammed, P.O.; Alsharif, A.M.; Guirao, J.L.G. New Chebyshev type inequalities via a general family of fractional integral operators with a modified Mittag-Leffler kernel. AIMS Math. 2021, 6, 11167-11186. [CrossRef]

22. Set, E.; Kashuri, A.; Mumcu, İ. Chebyshev type inequalities by using generalized proportional Hadamard fractional integrals via Polya-Szegö inequality with applications. Chaos Solitons Fractals 2021, 146, 110860. [CrossRef]

23. Özdemir, M.E.; Set, E.; Akdemir, A.O.; Sarikaya, M.Z. Some new Chebyshev type inequalities for functions whose derivatives belongs to $L_{p}$ spaces. Afr. Mat. 2015, 26, 1609-1619. [CrossRef]

24. Akdemir, A.O.; Butt, S.I.; Nadeem, M.; Ragusa, M.A. New general variants of Chebyshev type inequalities via generalized fractional integral operators. Mathematics 2021, 9, 122. [CrossRef]

25. Butt, S.I.; Nadeem, M.; Farid, G. On Caputo fractional derivatives via exponential s-convex functions. Turk. J. Sci. 2020, 5, 140-146

26. Alp, N.; Sarikaya, M.Z.; Kunt, M.; İşcan, İ. q-Hermite-Hadamard inequalities and quantum estimates for midpoint type inequalities via convex and quasi-convex functions. J. King Saud Univ.-Sci. 2018, 30, 193-203. [CrossRef]

27. İşcan, İ. Generalization of different type integral inequalities for s-convex functions via fractional integrals. Appl. Anal. 2014, 93, 1846-1862. [CrossRef]

28. Özcan, S. Hermite-Hadamard type inequalities for $m$-convex and $(\alpha, m)$-convex functions. J. Inequal. Appl. 2020, 2020, 10. [CrossRef]

29. İşcan, İ. A new generalization of some integral inequalities for $(\alpha, m)$-convex functions. Math. Sci. 2013, 7, 1-8. [CrossRef]

30. Liu, W.; Wen, W.; Park, J. Ostrowski type fractional integral inequalities for MT-convex functions. Miskolc Math. Notes 2015, 16, 249-256. [CrossRef]

31. Kashuri, A.; Liko, R. Some new Ostrowski type fractional integral inequalities for generalized $(r ; g, s, m, \varphi)$-preinvex functions via Caputo $k$-fractional derivatives. Int. J. Nonlinear Anal. Appl. 2017, 8, 109-124. [CrossRef]

32. Fernandez, A.; Mohammed, P.O. Hermite-Hadamard inequalities in fractional calculus defined using Mittag-Leffler kernels Math. Meth. Appl. Sci. 2021, 44, 8414-8431. [CrossRef]

33. Watson, G.N. A Treatise on the Theory of Bessel Functions; Cambridge University Press: Cambridge, UK, 1944.

34. Luke, Y.L. The Special Functions and Their Approximations; Academic Press: Cambridge, MA, USA, 1969; Volume I.

35. Kashuri, A.; Liko, R. Some new Hermite-Hadamard type inequalities and their applications. Stud. Sci. Math. Hung. 2019, 56, 103-142. [CrossRef]

36. Abdeljawad, T.; Mohammed, P.O.; Kashuri, A. New modified conformable fractional integral inequalities of Hermite-Hadamard type with applications. J. Funct. Spaces 2020, 2020, 4352357. [CrossRef]

37. Mohammed, P.O.; Abdeljawad, T.; Zeng, S.; Kashuri, A. Fractional Hermite-Hadamard integral inequalities for a new class of convex functions. Symmetry 2020, 12, 1485. [CrossRef]

38. Zhou, X.S.; Huang, C.X.; Hu, H.J.; Liu, L. Inequality estimates for the boundedness of multilinear singular and fractional integral operators. J. Inequal. Appl. 2013, 2013, 303. [CrossRef]

39. Kilbas, A.A.; Srivastava, H.M.; Trujillo, J.J. Theory and Applications of Fractional Differential Equations; North-Holland Mathematical Studies; Elsevier (North-Holland) Science Publishers: Amsterdam, The Netherlands; London, UK; New York, NY, USA, 2006; Volume 204

40. Baleanu, D.; Fernandez, A. On fractional operators and their classifications. Mathematics 2019, 7, 830. [CrossRef]

41. Srivastava, H.M.; Karlsson, P.W. Multiple Gaussian Hypergeometric Series; Halsted Press (Ellis Horwood Limited, Chichester): Chichester, UK; John Wiley and Sons: New York, NY, USA; Chichester, UK; Brisbane, Australia; Toronto, ON, Canada, 1985.

42. Barnett, N.S.; Cerone, P.; Dragomir, S.S.; Roumeliotis, J. Some inequalities for the dispersion of a random variable whose pdf is defined on a finite interval. J. Inequal. Pure Appl. Math. 2001, 2, 1-18.

43. Barnett, N.S.; Dragomir, S.S. Some elementary inequalities for the expectation and variance of a random variable whose pdf is defined on a finite interval. RGMIA Res. Rep. Colloq. 1999, 2, 1-7.

44. Cerone, P.; Dragomir, S.S. On some inequalities for the expectation and variance. Korean J. Comput. Appl. Math. 2000, 2, 357-380. [CrossRef]

45. Pečarič, J.E.; Proschan, F.; Tong, Y.L. Convex Functions, Partial Ordering and Statistical Applications; Academic Press: New York, NY, USA, 1991. 